Gianluigi Rozza, Martin Hess, Giovanni Stabile, Marco Tezzele, and Francesco Ballarin

\title{
1 Basic ideas and tools for projection-based model reduction of parametric partial differential equations
}

\begin{abstract}
We provide first the functional analysis background required for reducedorder modeling and present the underlying concepts of reduced basis model reduction. The projection-based model reduction framework under affinity assumptions, offline-online decomposition, and error estimation are introduced. Several tools for geometry parameterizations such as free form deformation, radial basis function interpolation, and inverse distance weighting interpolation are explained. The empirical interpolation method is introduced as a general tool to deal with nonaffine parameter dependency and nonlinear problems. The discrete and matrix versions of the empirical interpolation are considered as well. Active subspace properties are discussed to reduce high-dimensional parameter spaces as a preprocessing step. Several examples illustrate the methodologies.
\end{abstract}

Keywords: reduced basis method, radial basis function interpolation, shape morphing techniques, empirical interpolation method, active subspaces

MSC 2010: 65D99, 65J05, 65M15

\section{Introduction}

Parametric model order reduction (MOR) techniques have been developed in recent decades to deal with increasingly complex computational tasks. The ability to compute how quantities of interest change with respect to parameter variations provides insight and understanding, which is vital in all areas of science and engineering. Model reduction thus allows to deal with optimization or inverse problems of a whole new scale. Each chapter of the handbook gives an in-depth view of a MOR method,

Acknowledgement: We are grateful to the EU-COST European Union Cooperation in Science and Technology, section EU-MORNET Model Reduction Network, TD 1307 for pushing us into this initiative. This work is supported by European Union Funding for Research and Innovation - Horizon 2020 Program - in the framework of European Research Council Executive Agency: H2020 ERC CoG 2015 AROMA-CFD project 681447 “Advanced Reduced Order Methods with Applications in Computational Fluid Dynamics" to P. I. Gianluigi Rozza.

Gianluigi Rozza, Martin Hess, Giovanni Stabile, Marco Tezzele, Francesco Ballarin, Mathematics Area, SISSA mathLab, Trieste, Italy, e-mails: gianluigi.rozza@sissa.it,martin.hess@sissa.it, giovanni.stabile@sissa.it, marco.tezzele@sissa.it, francesco.ballarin@sissa.it

Ә Open Access. (C) 2021 Gianluigi Rozza et al., published by De Gruyter. (cc) BY-NC-ND This work is licensed under the Creative Commons Attribution-NonCommercial-NoDerivatives 4.0 International License. 
a particular application area, and analytical, numerical, or technical aspects of software frameworks for model reduction.

There exist a large number of MOR techniques used in many areas of science and engineering to improve computational performances and contain costs in a repetitive computational environment, such as many-query and real-time computing [93]. We assume a given parameterized partial differential equation (PDE) as starting point of the model reduction procedure. Typical parameters of interest are material coefficients, corresponding to physical qualities of the media which constitute the domain where the PDE is solved. Also a variable geometry can be of special interest in a task to find the optimal device configuration. Physical states such as the temperature might be considered an input parameter. It is a task of the mathematical modeling to identify the parameters of interest and how they enter the PDE. Once a parameterized model is identified, the MOR techniques described in this and the following chapters can be used either in a "black-box" fashion (nonintrusive way) or by intrusive means, which will be explained in detail, whenever this is necessary.

The particular numerical method to solve a PDE is most often not relevant to the model reduction procedure. We will therefore assume there is a numerical method available, which solves the problem to any required accuracy, and move seamlessly from the continuous form to the discretized form.

This chapter covers briefly the functional analysis framework relevant to many, but not all, MOR methods. Presented is the starting point of PDE-oriented MOR techniques, such as the POD method found in Chapter 2 of this volume, the PGD method found in Chapter 3 of this volume, the reduced basis method found in Chapter 4 of this volume, the hyperreduction technique found in Chapter 5 of this volume, the localized reduced-order modeling (ROM) found in Chapter 6 of this volume, and the data-driven methods found in Chapter 7 of this volume.

In particular, Section 1.1 provides what is needed for the projection-based ROM. Starting from the setting of the classical Lax-Milgram theorem for elliptic PDEs in Sections 1.1.1 and 1.1.2, a numerical discretization is introduced in Section 1.1.2.1. Due to brevity of representation, many concepts of functional analysis and theory of PDEs are only touched upon. Many references to the literature for further reading are given.

Projection-based ROM is presented in Section 1.1.3, with the following topics covered in detail: proper orthogonal decomposition (POD) in Section 1.1.3.1, the greedy algorithm in Section 1.1.3.2, the projection framework in Section 1.1.3.3, affine parameter dependency in Section 1.1.3.4, the offline-online decomposition in Section 1.1.3.6, and basic error estimation in Section 1.1.4.

Section 1.2 introduces efficient techniques for geometric parameterizations, arising from a reference domain approach, such as free form deformation (FFD) in Section 1.2.1, radial basis function (RBF) interpolation in Section 1.2.2, and inverse distance weighting (IDW) in Section 1.2.3.

A widely used method to generate an approximate affine parameter dependency is the empirical interpolation method (EIM). The original EIM is presented in Section 1.3 
as well as the discrete EIM in Section 1.3.3 and further options in Section 1.3.4. Several numerical examples show the use of the EIM in Section 1.3.5.

Section 1.4 introduces active subspaces as a preprocessing step to reduce the parameter space dimension. Corresponding examples are provided in Section 1.4.3 and also nonlinear dimensionality reduction is briefly discussed in Section 1.4.5.

A brief conclusion and an outlook of the handbook are given in Section 1.5.

\subsection{Basic notions and tools}

We briefly cover a few main results of linear functional analysis and the analysis of PDEs. This material serves as a reminder of the underlying concepts of model reduction but cannot replace a textbook on these subjects. For a more thorough background, we refer to the literature on functional analysis [30, 110], PDEs [1, 47, 82, 88], and numerical methods [2, 6, 29, 52, 80, 105].

\subsubsection{Parameterized partial differential equations}

Let $\Omega \subset \mathbb{R}^{d}$ denote a spatial domain in $d=1,2$, or 3 dimensions with boundary $\partial \Omega$. A Dirichlet boundary $\Gamma_{D} \subset \partial \Omega$ is given, where essential boundary conditions on the field of interest are prescribed. Introduce a Hilbert space $V(\Omega)$ equipped with inner product $(\cdot, \cdot)_{V}$ and induced norm $\|\cdot\|_{V}$. A Hilbert space $V(\Omega)$ is a function space, i. e., a function $u \in V(\Omega)$ is seen as a point in the vector space $V$, as is common in functional analysis. Each $u \in V(\Omega)$ defines a mapping $x \in \Omega \mapsto u(x) \in \mathbb{R}$ or $x \in \Omega \mapsto u(x) \in \mathbb{C}$, depending on whether a real or complex Hilbert space is considered. In many applications, $V$ is a subset of the Sobolev space $H^{1}(\Omega)$ as $V(\Omega)=\left\{v \in H^{1}(\Omega):\left.v\right|_{\Gamma_{D}}=0\right\}$. Vector-valued Hilbert spaces can be constructed using the Cartesian product of $V(\Omega)$. Given a parameter domain $\mathcal{P} \subset \mathbb{R}^{p}$, a particular parameter point is denoted by the $p$-tuple $\boldsymbol{\mu}=\left(\mu_{1}, \mu_{2}, \ldots, \mu_{p}\right)$. The set of all linear and continuous forms on $V$ defines the dual space $V^{\prime}$; let $L \in \mathcal{L}\left(V, V^{\prime}\right)$ denote a linear differential operator.

A field variable $u \in V: \Omega \rightarrow \mathbb{R}$ is defined implicitly as the solution to a parameterized linear PDE through the operator $L: V \times \mathcal{P} \rightarrow V^{\prime}$ with $L(\cdot ; \boldsymbol{\mu}) \in \mathcal{L}\left(V, V^{\prime}\right)$ and load vector $f_{L}(\boldsymbol{\mu}) \in V^{\prime}$ for each fixed $\boldsymbol{\mu}$, as

$$
L(u ; \boldsymbol{\mu})=f_{L}(\boldsymbol{\mu}) .
$$

As in the case of function spaces, operators between function spaces form vector spaces themselves, such as $L(\cdot ; \boldsymbol{\mu}) \in \mathcal{L}\left(V, V^{\prime}\right)$, with $\mathcal{L}\left(V, V^{\prime}\right)$ being the space of operators mapping from the vector space $V$ to $V^{\prime}$.

Typical examples of scalar-valued linear PDEs are the Poisson equation, the heat equation, and the wave equation, while typical examples of vector-valued linear PDEs 
are the Maxwell equations and the Stokes equations. The nonlinear case will be addressed in various chapters as well: Examples of nonlinear PDEs include the NavierStokes system and the equations describing nonlinear elasticity.

\subsubsection{Parameterized variational formulation}

The variational form or weak form of a parameterized linear PDE in the continuous setting is given as

$$
a(u(\boldsymbol{\mu}), v ; \boldsymbol{\mu})=f(v ; \boldsymbol{\mu}) \quad \forall v \in V,
$$

with bilinear form $a: V \times V \times \mathcal{P} \rightarrow \mathbb{R}$ and linear form $f: V \times \mathcal{P} \rightarrow \mathbb{R}$. In many application scenarios, a particular output of interest is sought, given by the linear form $l: V \times \mathcal{P} \rightarrow \mathbb{R}$ as

$$
s(\boldsymbol{\mu})=l(u(\boldsymbol{\mu}) ; \boldsymbol{\mu})
$$

In the case that $a(\cdot, \cdot ; \boldsymbol{\mu})$ is symmetric and $l=f$, the problem is called compliant. For each $\boldsymbol{\mu} \in \mathcal{P}$ assume coercivity and continuity of the bilinear form $a(\cdot, \cdot ; \boldsymbol{\mu})$, i. e.,

$$
\begin{aligned}
& a(w, w ; \boldsymbol{\mu}) \geq \alpha(\boldsymbol{\mu})\|w\|_{V}^{2}, \\
& a(w, v ; \boldsymbol{\mu}) \leq \gamma(\boldsymbol{\mu})\|w\|_{V}\|v\|_{V},
\end{aligned}
$$

and continuity of the linear form $f(\cdot ; \boldsymbol{\mu})$,

$$
f(w ; \boldsymbol{\mu}) \leq \delta(\boldsymbol{\mu})\|w\|_{V},
$$

with parameter-independent bounds, which satisfy $0<\alpha \leq \alpha(\boldsymbol{\mu}), \gamma(\boldsymbol{\mu}) \leq \gamma<\infty$, and $\delta(\boldsymbol{\mu}) \leq \delta<\infty$. To do actual computations, the bilinear form is discretized into a linear equation. The coercivity property means that the matrix discretizing the bilinear form will be positive definite.

For fixed parameter the well-posedness of (1.2) is then established by the LaxMilgram theorem.

Theorem 1.1 (Lax-Milgram theorem). Let $a: V \times V \rightarrow \mathbb{R}$ be a continuous and coercive bilinear form over a Hilbert space $V$ and $f \in V^{\prime}$ a continuous linear form. Then the variational problem

$$
a(u, v)=f(v) \quad \forall v \in V
$$

has a unique solution $u \in V$ and we have

$$
\|u\|_{V} \leq \frac{1}{\alpha}\|f\|_{V},
$$

with the coercivity constant $\alpha>0$ of the bilinear form. 
Thus, in the parametric setting, the $\boldsymbol{\mu}$-dependence also carries over to the coercivity constant as $\alpha=\alpha(\boldsymbol{\mu})$.

The function space in which the field variable resides is called the ansatz space, while the second function space is called the test space, i. e., where a test function $v$ resides. If the test space is distinct from the ansatz space, then the bilinear form is defined over $a: V \times W \times \mathcal{P} \rightarrow \mathbb{R}$ for $V$ and $W$ Hilbert spaces. With $f \in W^{\prime}$ and for fixed $\boldsymbol{\mu}$, the well-posedness is then established through the Banach-Nečas-Babuška theorem.

Theorem 1.2 (Banach-Nečas-Babuška theorem). Let $V$ and $W$ denote Hilbert spaces, let $a: V \times W \rightarrow \mathbb{R}$ be a continuous bilinear form, and $f \in W^{\prime}$. Then the variational problem

$$
a(u, v)=f(v) \quad \forall v \in W
$$

has a unique solution if and only if

(i) the inf-sup condition holds, i.e.,

$$
\exists \beta>0, \text { s.t., } \beta \leq \inf _{v \in V \backslash\{0\}} \sup _{w \in W \backslash\{0\}} \frac{a(v, w)}{\|v\|_{V}\|w\|_{W}},
$$

(ii) $\forall w \in W$ :

$$
\{a(v, w)=0 \forall v \in V\} \Longrightarrow w=0 .
$$

\subsubsection{Discretized parameterized variational formulation}

The method of weighted residuals is used to cast (1.1) into a discrete variational formulation. Given the linear PDE $L(u ; \boldsymbol{\mu})=f_{L}(\boldsymbol{\mu})$, consider a discrete, i. e., finitedimensional, approximation $u_{h} \in V_{h} \subset V$ to $u$ as

$$
u_{h}(\boldsymbol{\mu})=\sum_{i=1}^{N_{h}} u_{h}^{(i)} \varphi^{i}
$$

The dimension of $V_{h}$ is $N_{h}$ and the set of ansatz functions $\varphi^{i}(\mathbf{x}): \Omega \rightarrow \mathbb{R}$ belong to $V$. The $u_{h}^{(i)}$ are scalar coefficients such that the vector $\mathbf{u}_{h}=\left(u_{h}^{(1)}, \ldots, u_{h}^{\left(N_{h}\right)}\right)^{T} \in \mathbb{R}^{N_{h}}$ is the coordinate representation of $u_{h}$ in the basis $\left\{\varphi^{i}\right\}$ of $V_{h}$. A conforming discretization is considered, i. e., $V_{h} \subset V$ holds.

Plugging (1.10) into (1.1) yields the discrete residual $R\left(u_{h}(\boldsymbol{\mu})\right)=L\left(u_{h}(\boldsymbol{\mu}) ; \boldsymbol{\mu}\right)-$ $f_{L}(\boldsymbol{\mu}) \in V^{\prime}$. To compute the scalar coefficients $u_{h}^{(i)}$, Galerkin orthogonality is invoked, as

$$
0=\left(\varphi_{j}, R\right)_{\left(V, V^{\prime}\right)}, \quad j=1 \ldots N_{h},
$$

where $(\cdot, \cdot)_{\left(V, V^{\prime}\right)}$ is the duality pairing between $V$ and $V^{\prime}$. 
In short, Galerkin orthogonality means that the test space is orthogonal to the residual. In Ritz-Galerkin methods, the residual is tested against the same set of functions as the ansatz functions. If test space and trial space are different, one speaks of a Petrov-Galerkin method. Numerous discretization methods can be understood in terms of the method of weighted residuals. They are distinguished by the particular choice of trial and test space.

The well-posedness of the discrete setting follows the presentation of the continuous setting, by casting the equations and properties over $V_{h}$ instead of $V$.

The weak form in the discrete setting is given as

$$
a\left(u_{h}(\boldsymbol{\mu}), v_{h} ; \boldsymbol{\mu}\right)=f\left(v_{h} ; \boldsymbol{\mu}\right) \quad \forall v_{h} \in V_{h}
$$

with bilinear form $a: V_{h} \times V_{h} \times \mathcal{P} \rightarrow \mathbb{R}$ and linear form $f: V_{h} \times \mathcal{P} \rightarrow \mathbb{R}$. The discrete bilinear form is then derived from (1.11) through the integration-by-parts formula and Green's theorem.

Correspondingly, the discrete coercivity constant $\alpha_{h}(\mu)$ and the discrete continuity constant $\gamma_{h}(\mu)$ are defined as

$$
\begin{aligned}
& \alpha_{h}(\boldsymbol{\mu})=\min _{w_{h} \in V_{h}} \frac{a\left(w_{h}, w_{h} ; \boldsymbol{\mu}\right)}{\left\|w_{h}\right\|_{V_{h}}^{2}}, \\
& \gamma_{h}(\boldsymbol{\mu})=\max _{w_{h} \in V_{h}} \max _{v_{h} \in V_{h}} \frac{a\left(w_{h}, v_{h} ; \boldsymbol{\mu}\right)}{\left\|w_{h}\right\|_{V_{h}}\left\|v_{h}\right\|_{V_{h}}} .
\end{aligned}
$$

The well-posedness of (1.2) is then analogously established by the Lax-Milgram theorem and the Banach-Nečas-Babuška theorem. Cea's lemma is a fundamental result about the approximation quality that can be achieved.

Lemma 1.3 (Cea's lemma). Let $a: V \times V \rightarrow \mathbb{R}$ be a continuous and coercive bilinear form over a Hilbert space $V$ and $f \in V^{\prime}$ a continuous linear form. Given a conforming finite-dimensional subspace $V_{h} \subset V$, the continuity constant $\gamma$, and coercivity constant $\alpha$ of $a(\cdot, \cdot)$, for the solution $u_{h}$ to

$$
a\left(u_{h}, v_{h}\right)=f\left(v_{h}\right) \quad \forall v_{h} \in V_{h},
$$

we have

$$
\left\|u-u_{h}\right\|_{V} \leq \frac{\gamma}{\alpha} \inf _{v_{h} \in V_{h}}\left\|u-v_{h}\right\|_{V}
$$

The stiffness matrix $\mathbb{A}_{h} \in \mathbb{R}^{N_{h} \times N_{h}}$ assembles the bilinear form entrywise as $\left(\mathbb{A}_{h}\right)_{i j}=$ $a\left(\varphi^{j}, \varphi^{i}\right)$. The load vector $\mathbf{f}_{h} \in \mathbb{R}^{N_{h}}$ is assembled entrywise as $\left(\mathbf{f}_{h}\right)_{i}=f\left(\varphi^{i}\right)$ and the solution vector is denoted $\mathbf{u}_{h}$ with coefficients $u_{h}^{(j)}$.

Then solving (1.12) amounts to solving the linear system

$$
\mathbb{A}_{h} \mathbf{u}_{h}=\mathbf{f}_{h} .
$$


The most common discretization method is the finite element method [13], besides the finite difference [97], discontinuous Galerkin [4], finite volume [48], and spectral element methods [19].

\subsubsection{Model reduction basic concepts}

A wide variety of ROM methods exist today, thanks to large research efforts in the last decades. Reduced basis MOR is a projection-based MOR method and also shares many features with other MOR methods, so that the topics mentioned here will occur throughout the handbook. Two common algorithms for the generation of a projection space, POD and the greedy algorithm, are presented first.

\subsubsection{Proper orthogonal decomposition}

Assume a sampled set of high-fidelity solutions $\left\{u_{h}\left(\boldsymbol{\mu}_{i}\right), i=1, \ldots, N_{\max }\right\}$, i. e., solutions to (1.12) or (1.17), respectively. The discrete solution vectors are stored columnwise in a snapshot matrix $\mathbb{S} \in \mathbb{R}^{N_{h} \times N_{\max }}$. POD compresses the data stored in $\mathbb{S}$ by computing an orthogonal matrix $\mathbb{V}$, which is a best approximation in the least-squares sense to $\mathbb{S}$. In particular, the POD solution of size $N$ is the solution to

$$
\begin{array}{r}
\min _{\mathbb{V} \in \mathbb{R}^{N_{h} \times N}}\left\|\mathbb{S}-\mathbb{V} \mathbb{V}^{T} \mathbb{S}\right\|_{F}, \\
\text { subject to } \mathbb{V}^{T} \mathbb{V}=\mathbb{I}_{N \times N},
\end{array}
$$

with $\|\cdot\|_{F}$ being the Frobenius norm and $\mathbb{I}_{N \times N}$ being the identity matrix.

There exists a solution to (1.18)-(1.19) according to the Eckardt-Young-Mirsky theorem [43], which can be computed with singular value decomposition (SVD) as

$$
\mathbb{S}=\mathbb{U} \Sigma \mathbb{Z}
$$

with orthogonal matrix $\mathbb{U} \in \mathbb{R}^{N_{h} \times N_{h}}$, rectangular diagonal matrix $\Sigma \in \mathbb{R}^{N_{h} \times N_{\max }}$, and orthogonal matrix $\mathbb{Z} \in \mathbb{R}^{N_{\max } \times N_{\max }}$. The solution $\mathbb{V}$ is composed of the first $N$ column vectors of $\mathbb{U}$. They are also called the POD modes. The diagonal entries $\left\{\sigma_{i}, i=\right.$ $\left.1, \ldots, \min \left(N_{h}, N_{\max }\right)\right\}$ of $\Sigma$ are nonnegative and are called singular values. We have

$$
\min _{\mathbb{V} \in \mathbb{R}^{N_{h} \times N}}\left\|\mathbb{S}-\mathbb{V}^{T} \mathbb{S}\right\|_{F}=\sum_{i=N+1}^{\min \left(N_{h}, N_{\max }\right)} \sigma_{i} .
$$

Thus, the neglected singular values give an indication of the approximate truncation error. In practise, a high tolerance threshold like $99 \%$ or $99.99 \%$ is chosen and $N$ is determined so that the sum of the first $N$ singular values reaches this percentage of the sum of all singular values. In many applications, an exponential singular value decay can be observed, which allows to reach the tolerance with a few POD modes. 


\subsubsection{Greedy algorithm}

The greedy algorithm also computes an orthogonal matrix $\mathbb{V} \in \mathbb{R}^{N_{h} \times N}$ to serve as a projection operator, just as in the POD case. The greedy algorithm is an iterative procedure, which enriches the snapshot space according to where an error indicator or error estimator $\Delta$ attains its maximum. Starting from a field solution at a given initial parameter value, the parameter location is sought, whose field solution is worst approximated with the initial solution. This solution is then computed and appended to the projection matrix to obtain a two-dimensional projection space. The greedy typically searches for new snapshot solutions within a discrete surrogate $P$ of the parameter space $\mathcal{P}$. The process is repeated until a given tolerance on the error estimator is fulfilled. The error estimator is residual-based and estimates the error between a reducedorder solve for a projection space $\mathbb{V}$ and the high-fidelity solution (Section 1.1.4). The greedy algorithm is stated in pseudo-code in Algorithm 1.1.

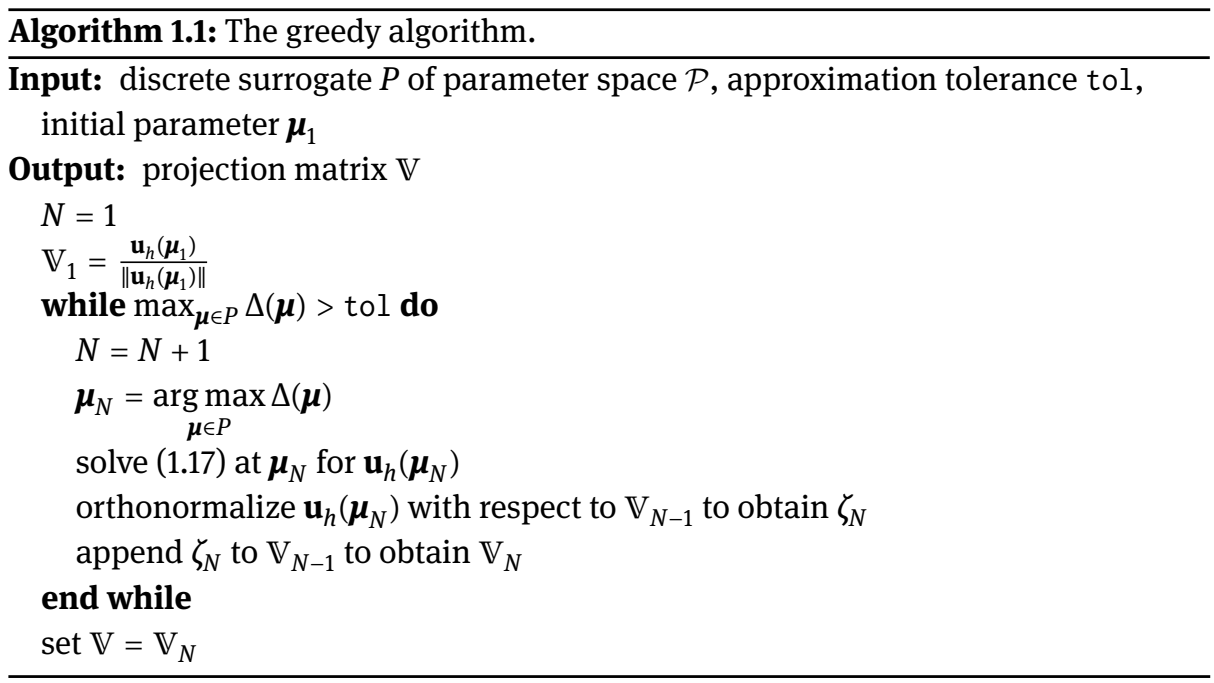

\subsubsection{Reduced-order system}

Starting from the discrete high-fidelity formulation (1.12), another Galerkin projection is invoked to arrive at the reduced-order formulation. Assume a projection space $V_{N}$ is then determined through either a POD or the greedy sampling, with $\mathbb{V} \in \mathbb{R}^{N_{h} \times N}$ denoting a discrete basis of $V_{N}$. Thus $V_{N} \subset V_{h}$ and $\operatorname{dim} V_{N}=N$.

The reduced-order variational formulation is to determine $u_{N}(\boldsymbol{\mu}) \in V_{N}$, such that

$$
a\left(u_{N}(\boldsymbol{\mu}), v_{N} ; \boldsymbol{\mu}\right)=f\left(v_{N} ; \boldsymbol{\mu}\right) \quad \forall v_{N} \in V_{N}
$$


Equation (1.17) is then projected onto the reduced-order space as

$$
\mathbb{V}^{T} \mathbb{A}_{h} \mathbb{V} \mathbf{u}_{N}=\mathbb{V}^{T} \mathbf{f}_{h}
$$

The reduced system matrix $\mathbb{A}_{N}=\mathbb{V}^{T} \mathbb{A}_{h} \mathbb{V}$ is then a dense matrix of small size $N \times N$ as depicted in (1.24):

$$
\left[\mathbb{A}_{N}\right]=[\mathbb{V}]^{T}\left[\begin{array}{ccc}
a\left(\varphi^{1}, \varphi^{1}\right) & \ldots & \ldots \\
\ldots & \ldots & \ldots \\
\ldots & \ldots & a\left(\varphi^{N_{h}}, \varphi^{N_{h}}\right)
\end{array}\right]\left[\begin{array}{l}
\mathbb{V} \\
\end{array}\right]
$$

The high-order solution is then approximated as

$$
\mathbf{u}_{h} \approx \mathbb{V} \mathbf{u}_{N}
$$

\subsubsection{Affine parameter dependency}

Many MOR algorithms rely on an affine parameter dependency, because the affine parameter dependency provides the computational efficiency of the model reduction. Thus, it is a significant advancement from the 2000s [85] over the first use of ROMs [3, 76].

An affine parameter dependency means that the bilinear form can be expanded as

$$
a(\cdot, \cdot ; \boldsymbol{\mu})=\sum_{i=1}^{Q_{a}} \Theta_{a}^{i}(\boldsymbol{\mu}) a_{i}(\cdot, \cdot)
$$

and affine expansions hold as

$$
\begin{gathered}
f(\cdot ; \boldsymbol{\mu})=\sum_{i=1}^{Q_{f}} \Theta_{f}^{i}(\boldsymbol{\mu}) f_{i}(\cdot), \\
l(\cdot ; \boldsymbol{\mu})=\sum_{i=1}^{Q_{l}} \Theta_{l}^{i}(\boldsymbol{\mu}) l_{i}(\cdot),
\end{gathered}
$$

with scalar-valued functions $\Theta_{a}^{i}: \mathcal{P} \rightarrow \mathbb{R}, \Theta_{f}^{i}: \mathcal{P} \rightarrow \mathbb{R}$, and $\Theta_{l}^{i}: \mathcal{P} \rightarrow \mathbb{R}$.

Correspondingly the linear system (1.17) can be expanded as

$$
\left(\sum_{i=1}^{Q_{a}} \Theta_{a}^{i}(\boldsymbol{\mu}) \mathbb{A}_{i}\right) \mathbf{u}_{h}=\sum_{i=1}^{Q_{f}} \Theta_{f}^{i}(\boldsymbol{\mu}) \mathbf{f}_{i}
$$

as well as the reduced-order form (1.23)

$$
\mathbb{V}^{T}\left(\sum_{i=1}^{Q_{a}} \Theta_{a}^{i}(\boldsymbol{\mu}) \mathbb{A}_{i}\right) \mathbb{V} \mathbf{u}_{N}=\mathbb{V}^{T} \sum_{i=1}^{Q_{f}} \Theta_{f}^{i}(\boldsymbol{\mu}) \mathbf{f}_{i},
$$




$$
\left(\sum_{i=1}^{Q_{a}} \Theta_{a}^{i}(\boldsymbol{\mu}) \mathbb{V}^{T} \mathbb{A}_{i} \mathbb{V}\right) \mathbf{u}_{N}=\sum_{i=1}^{Q_{f}} \Theta_{f}^{i}(\boldsymbol{\mu}) \mathbb{V}^{T} \mathbf{f}_{i}
$$

MOR relies on an affine parameter dependency, such that all computations depending on the high-order model size can be moved into a parameter-independent offline phase, while having a fast input-output evaluation online. If the problem is not affine, an affine representation can be approximated using a technique such as the EIM (Section 1.3).

\subsubsection{Affine shape parameterizations: an example}

Consider heat conduction in a square domain $\Omega(x, y)=[0,1]^{2}$. On the left side $x=0$, inhomogeneous Neumann conditions, i. e., a nonzero heat flux, are imposed and on the right side $x=1$, homogeneous Dirichlet conditions, i.e., zero temperature, are imposed. On the top and bottom sides, homogeneous Neumann conditions, i. e., a zero heat flux, are imposed. Consider two different media with different conductivities $\sigma_{1}$ and $\sigma_{2}$ occupying the subdomains $\Omega_{1}(\mu)=[0, \mu] \times[0,1]$ and $\Omega_{2}(\mu)=[\mu, 1] \times[0,1]$, for $\mu \in \mathcal{P}=(0,1)$, as shown in Figure 1.1. For the sake of clarity, in the rest of this section we identify the one-dimensional parameter vector $\boldsymbol{\mu}$ with its (only) component $\mu$, thus dropping the bold notation from the symbol.

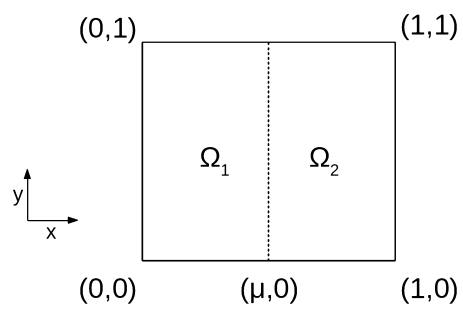

Figure 1.1: The computational domain is subdivided into two domains $\Omega=\Omega_{1} \cup \Omega_{2}$, depending on the parameter $\mu$. Shown here for $\mu=0.5$.

Choosing $\bar{\mu}=0.5$ as the reference configuration, there exist affine transformations from the reference domain to the actual domain. We have

$$
\begin{aligned}
& T_{1}: \Omega_{1}(\bar{\mu}) \rightarrow \Omega_{1}(\mu):(\bar{x}, \bar{y}) \mapsto(2 \mu \bar{x}, \bar{y}), \\
& T_{2}: \Omega_{2}(\bar{\mu}) \rightarrow \Omega_{2}(\mu):(\bar{x}, \bar{y}) \mapsto((2-2 \mu) \bar{x}, \bar{y})+(2 \mu-1,0) .
\end{aligned}
$$

In general, an affine transformation of a subdomain can be expressed as

$$
T_{k}: \Omega_{k}(\bar{\mu}) \rightarrow \Omega_{k}(\mu): \mathbf{x} \mapsto G_{k}(\mu) \mathbf{x}+D_{k}(\mu),
$$

with $\mathbf{x} \in \mathbb{R}^{d}, G_{k} \in \mathbb{R}^{d \times d}$ and $D_{k} \in \mathbb{R}^{d}$ in $d=2,3$ spatial dimensions. 
Thus, the bilinear form

$$
a(u, v ; \mu)=\int_{\Omega_{1}(\mu)} \sigma_{1} \nabla u \cdot \nabla v d \mathbf{x}+\int_{\Omega_{2}(\mu)} \sigma_{2} \nabla u \cdot \nabla v d \mathbf{x}
$$

can be mapped to the reference domain with the inverse affine transformation

$$
T_{k}^{-1}: \Omega_{k}(\mu) \rightarrow \Omega_{k}(\bar{\mu}): \mathbf{x} \mapsto G_{k}^{-1}(\mu) \mathbf{x}-G_{k}^{-1}(\mu) D_{k}(\mu),
$$

and integration by substitution as

$$
\begin{aligned}
a(u, v ; \mu)= & \int_{\Omega_{1}(\bar{\mu})} \sigma_{1}\left(\nabla u G_{1}^{-1}(\mu)\right) \cdot\left(G_{1}^{-T}(\mu) \nabla v\right) \operatorname{det}\left(G_{1}(\mu)\right) d \mathbf{x} \\
& +\int_{\Omega_{2}(\bar{\mu})} \sigma_{2}\left(\nabla u G_{2}^{-1}(\mu)\right) \cdot\left(G_{2}^{-T}(\mu) \nabla v\right) \operatorname{det}\left(G_{2}(\mu)\right) d \mathbf{x},
\end{aligned}
$$

which establishes the affine parameter dependency (1.26) by computing $\Theta_{a}^{i}(\mu)$ from the coefficients of $G_{1}$ and $G_{2}[85,84,28]$. That is,

$$
\begin{aligned}
& \int_{\Omega_{1}(\bar{\mu})} \sigma_{1}\left(\nabla u G_{1}^{-1}(\mu)\right) \cdot\left(G_{1}^{-T}(\mu) \nabla v\right) \operatorname{det}\left(G_{1}(\mu)\right) d \mathbf{x} \\
& =\int_{\Omega_{1}(\bar{\mu})} \sigma_{1}\left((2 \mu)^{-1} \partial_{x} u, \partial_{y} u\right) \cdot\left((2 \mu)^{-1} \partial_{x} v, \partial_{y} v\right) 2 \mu d \mathbf{x} \\
& =(2 \mu)^{-1} \int_{\Omega_{1}(\bar{\mu})} \sigma_{1}\left(\partial_{x} u\right)\left(\partial_{x} v\right) d \mathbf{x}+2 \mu \int_{\Omega_{1}(\bar{\mu})} \sigma_{1}\left(\partial_{y} u\right)\left(\partial_{y} v\right) d \mathbf{x},
\end{aligned}
$$

and

$$
\begin{aligned}
& \int_{\Omega_{2}(\bar{\mu})} \sigma_{2}\left(\nabla u G_{2}^{-1}(\mu)\right) \cdot\left(G_{2}^{-T}(\mu) \nabla v\right) \operatorname{det}\left(G_{2}(\mu)\right) d \mathbf{x} \\
& =\int_{\Omega_{2}(\bar{\mu})} \sigma_{2}\left((2-2 \mu)^{-1} \partial_{x} u, \partial_{y} u\right) \cdot\left((2-2 \mu)^{-1} \partial_{x} v, \partial_{y} v\right)(2-2 \mu) d \mathbf{x} \\
& =(2-2 \mu)^{-1} \int_{\Omega_{2}(\bar{\mu})} \sigma_{2}\left(\partial_{x} u\right)\left(\partial_{x} v\right) d \mathbf{x}+(2-2 \mu) \int_{\Omega_{2}(\bar{\mu})} \sigma_{2}\left(\partial_{y} u\right)\left(\partial_{y} v\right) d \mathbf{x},
\end{aligned}
$$

which establishes the affine form (1.26) with $Q_{a}=4$, and

$$
\begin{aligned}
& \Theta_{a}^{1}(\mu)=(2 \mu)^{-1}, \\
& \Theta_{a}^{2}(\mu)=2 \mu, \\
& \Theta_{a}^{3}(\mu)=(2-2 \mu)^{-1},
\end{aligned}
$$




$$
\Theta_{a}^{4}(\mu)=2-2 \mu
$$

and

$$
\begin{aligned}
& a_{1}(\cdot, \cdot)=\int_{\Omega_{1}(\bar{\mu})} \sigma_{1}\left(\partial_{x} u\right)\left(\partial_{x} v\right) d \mathbf{x}, \\
& a_{2}(\cdot, \cdot)=\int_{\Omega_{1}(\bar{\mu})} \sigma_{1}\left(\partial_{y} u\right)\left(\partial_{y} v\right) d \mathbf{x}, \\
& a_{3}(\cdot, \cdot)=\int_{\Omega_{2}(\bar{\mu})} \sigma_{2}\left(\partial_{x} u\right)\left(\partial_{x} v\right) d \mathbf{x}, \\
& a_{4}(\cdot, \cdot)=\int_{\Omega_{2}(\bar{\mu})} \sigma_{2}\left(\partial_{y} u\right)\left(\partial_{y} v\right) d \mathbf{x} .
\end{aligned}
$$

The second and fourth terms can be further simplified to a term depending on $2 \mu$ and a $\mu$-independent term, but in this case it still leaves $Q_{a}=4$ terms. In some cases the number of affine terms can be automatically reduced further using symbolic computations.

\subsubsection{Offline-online decomposition}

The offline-online decomposition enables the computational speedup of the ROM approach in many-query scenarios. It is also known as the offline-online paradigm, which assumes that a computation-intensive offline phase can be performed on a supercomputer, which generates all quantities depending on the large discretization size $N_{h}$. Once completed, a reduced-order solve, i. e., an online solve for a new parameter of interest, can be performed with computational cost independent of the large discretization size $N_{h}$. The online phase can thus be performed even on mobile and embedded devices (Figure 1.2). If a supercomputer is not available, this can be relaxed, however. There exist heuristic algorithms to make also the offline phase feasible on a common workstation, such that a typical scenario would be that the offline phase runs overnight and a reduced model is available the next morning.

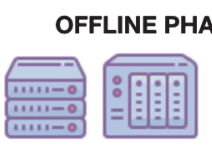

HPC cluster

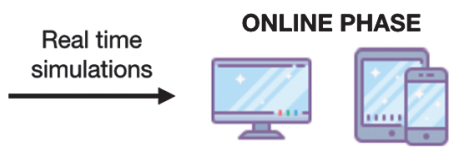

Portable devices

Figure 1.2: Offline-online paradigm. The complex high-fidelity simulations are carried out in high performance clusters (HPCs) for given preselected parameters. The solution snapshots can be stored and the ROM trained. Then in the offline phase the ROM provides approximated solutions at new untried parameters in real-time on simple portable devices. 
Noting that the terms $\mathbb{V}^{T} \mathbb{A}_{i} \mathbb{V}$ and $\mathbb{V}^{T} \mathbf{f}_{i}$ in (1.31) are parameter-independent, they can be precomputed, prior to any ROM parameter sweep. This will store small-sized dense matrices of dimension $N \times N$. Once a reduced-order solution $\mathbf{u}_{N}$ is desired for a given parameter $\boldsymbol{\mu}$, the sum given in (1.31) is formed and solved for $\mathbf{u}_{N}$. Since this is the same as solving (1.23), the reduced-order approximation is then available as $\mathbf{u}_{h} \approx \mathbb{V} \mathbf{u}_{N}$; see (1.25).

\subsubsection{Error bounds}

In this section we develop effective and reliable a posteriori error estimators for the field variable or an output of interest. The use of such error bounds drives the construction of the reduced basis during the offline stage, thanks to the so-called greedy algorithm. Moreover, during the online stage, such bounds provide a certified accuracy of the proposed ROM.

Following [85], we introduce residual-based a posteriori error estimation for the elliptic case. From (1.12) and (1.22) it follows that the error $e(\boldsymbol{\mu})=u_{h}(\boldsymbol{\mu})-u_{N}(\boldsymbol{\mu})$ satisfies

$$
a\left(e(\boldsymbol{\mu}), v_{h} ; \boldsymbol{\mu}\right)=r\left(v_{h} ; \boldsymbol{\mu}\right) \quad \forall v_{h} \in V_{h},
$$

where the residual $r(\cdot ; \boldsymbol{\mu}) \in V_{h}^{\prime}$ is defined as

$$
r\left(v_{h} ; \boldsymbol{\mu}\right)=f\left(v_{h} ; \boldsymbol{\mu}\right)-a\left(u_{N}(\boldsymbol{\mu}), v_{h} ; \boldsymbol{\mu}\right) \quad \forall v_{h} \in V_{h} .
$$

The following theorem further characterizes the relation between error and residual:

Theorem 1.4. Under compliance assumptions, the following inequalities hold:

$$
\begin{aligned}
& \|e(\boldsymbol{\mu})\|_{\boldsymbol{\mu}}=\left\|u_{h}(\boldsymbol{\mu})-u_{N}(\boldsymbol{\mu})\right\|_{\boldsymbol{\mu}} \leq \Delta_{e n}(\boldsymbol{\mu})=\frac{\|r(\cdot ; \boldsymbol{\mu})\|_{V_{h}^{\prime}}}{\sqrt{\alpha_{h}(\boldsymbol{\mu})}}, \\
& 0 \leq s_{h}(\boldsymbol{\mu})-s_{N}(\boldsymbol{\mu}) \leq \Delta_{s}(\boldsymbol{\mu})=\frac{\|r(\cdot ; \boldsymbol{\mu})\|_{V_{h}^{\prime}}^{2}}{\alpha_{h}(\boldsymbol{\mu})}
\end{aligned}
$$

where $\|v\|_{\boldsymbol{\mu}}^{2}=a(v, v ; \boldsymbol{\mu})$ defines an equivalent norm to $\|v\|_{V_{h}}$.

Proof. The norm $\|\cdot\|_{\boldsymbol{\mu}}$ defines an equivalent norm thanks to symmetry, continuity, and coercivity of $a(\cdot, \cdot ; \boldsymbol{\mu})$.

Since $e(\boldsymbol{\mu}) \in V_{h}$, from (1.53) with $v_{h}=e(\boldsymbol{\mu})$ it follows that

$$
\|e(\boldsymbol{\mu})\|_{\boldsymbol{\mu}}^{2}=a(e(\boldsymbol{\mu}), e(\boldsymbol{\mu}) ; \boldsymbol{\mu})=r(e(\boldsymbol{\mu}) ; \boldsymbol{\mu}) \leq\|r(\cdot ; \boldsymbol{\mu})\|_{V_{h}^{\prime}}\|e(\boldsymbol{\mu})\|_{V_{h}},
$$

the last inequality being due to the definition of the norm in $V_{h}^{\prime}$. Furthermore, due to coercivity, we have

$$
\|e(\boldsymbol{\mu})\|_{\boldsymbol{\mu}}^{2}=a(e(\boldsymbol{\mu}), e(\boldsymbol{\mu}) ; \boldsymbol{\mu}) \geq \alpha(\boldsymbol{\mu})\|e(\boldsymbol{\mu})\|_{V_{h}}^{2}
$$


Combining these two results yields (1.55).

Furthermore, since $l=f$ are linear forms,

$$
s_{h}(\boldsymbol{\mu})-s_{N}(\boldsymbol{\mu})=l(e(\boldsymbol{\mu}) ; \boldsymbol{\mu})=f(e(\boldsymbol{\mu}) ; \boldsymbol{\mu})=a\left(u_{h}(\boldsymbol{\mu}), e(\boldsymbol{\mu}) ; \boldsymbol{\mu}\right) .
$$

From (1.53) with $v_{h}:=v_{N} \in V_{N}$ and (1.22) it follows that

$$
a\left(e(\boldsymbol{\mu}), v_{N} ; \boldsymbol{\mu}\right)=r\left(v_{N}(\boldsymbol{\mu}) ; \boldsymbol{\mu}\right)=0 .
$$

This holds in particular for $v_{N}=u_{N}(\boldsymbol{\mu})$. Moreover, due to symmetry,

$$
a\left(u_{N}(\boldsymbol{\mu}), e(\boldsymbol{\mu}) ; \boldsymbol{\mu}\right)=0
$$

as well. Thus, $a\left(u_{h}(\boldsymbol{\mu}), e(\boldsymbol{\mu}) ; \boldsymbol{\mu}\right)=a(e(\boldsymbol{\mu}), e(\boldsymbol{\mu}) ; \boldsymbol{\mu})$ in (1.57), and we conclude that

$$
s_{h}(\boldsymbol{\mu})-s_{N}(\boldsymbol{\mu})=\|e(\boldsymbol{\mu})\|_{\boldsymbol{\mu}}^{2}
$$

The upper bound in (1.56) is then a consequence of (1.55), while the lower bound trivially holds as the right-hand side of (1.58) is a nonnegative quantity.

Offline-online decomposition is usually solicited for the a posteriori error bounds introduced by the previous theorem, for the sake of a fast computation of the righthand side of (1.55)-(1.56). This requires the efficient evaluation of both the numerator (dual norm of the residual) and the denominator (parameterized coercivity constant). The Riesz representation theorem is employed to define the unique $\hat{r}(\boldsymbol{\mu}) \in V_{h}$ such that

$$
\left(\hat{r}(\boldsymbol{\mu}), v_{h}\right)_{V_{h}}=r\left(v_{h} ; \boldsymbol{\mu}\right), \quad \forall v_{h} \in V_{h}
$$

Under affine separability assumptions (1.26)-(1.28), we have

$$
r\left(v_{h} ; \boldsymbol{\mu}\right)=\sum_{i=1}^{Q_{f}} \Theta_{f}^{i}(\boldsymbol{\mu}) f_{i}\left(v_{h}\right)-\sum_{n=1}^{N} \mathbf{u}_{N n} \sum_{i=1}^{Q_{a}} \Theta_{a}^{i}(\boldsymbol{\mu}) a_{i}\left(\zeta^{n}, v_{h}\right), \quad \forall v_{h} \in V_{h},
$$

so that an affine expansion with $Q_{f}+N Q_{a}$ terms is obtained for $r(\cdot ; \boldsymbol{\mu})$. Riesz representation is then invoked for

$$
\begin{array}{lll}
r_{1}\left(v_{h} ; \boldsymbol{\mu}\right)=f_{1}\left(v_{h}\right), & \ldots, & r_{Q_{f}}\left(v_{h} ; \boldsymbol{\mu}\right)=f_{Q_{f}}\left(v_{h}\right), \\
r_{Q_{f}+1}\left(v_{h} ; \boldsymbol{\mu}\right)=a_{1}\left(\zeta^{1}, v_{h}\right), & \ldots, & r_{Q_{f}+Q_{a}}\left(v_{h} ; \boldsymbol{\mu}\right)=a_{Q_{a}}\left(\zeta^{1}, v_{h}\right), \\
\ldots & & \\
r_{Q_{f}+(N-1) Q_{a}+1}\left(v_{h} ; \boldsymbol{\mu}\right)=a_{1}\left(\zeta^{N}, v_{h}\right), & \ldots, & r_{Q_{f}+N Q_{a}}\left(v_{h} ; \boldsymbol{\mu}\right)=a_{Q_{a}}\left(\zeta^{N}, v_{h}\right)
\end{array}
$$

during the offline stage, storing the corresponding solutions to (1.59).

As concerns the evaluation of the denominator of (1.55)-(1.56), exact evaluation of $\alpha(\boldsymbol{\mu})$ is seldom employed. Instead, an offline-online decomposable lower bound is 
sought. Early proposals on the topic are available in $[107,78,106,85,18]$. In 2007, the successive constraint method (SCM) was devised in [57] based on successive linear programming approximations, and subsequently extended in [26, 27, 103, 111]. Alternative methodologies based on interpolation techniques have also appeared in recent years in $[54,71,59]$.

A posteriori error estimation can be derived for more general problems as well (including noncoercive linear, nonlinear, or time-dependent problems), through application of the Brezzi-Rappaz-Raviart theory. We refer to [106, 41, 109, 70, 81] for a few representative cases. To this end, extensions of SCM are discussed in [27, 55, 58, 25].

\subsection{Geometrical parameterization for shapes and domains}

In this section we discuss problems characterized by a geometrical parameterization. In particular, a reference domain approach is discussed, relying on a map that deforms the reference domain into the parameterized one. Indeed, while affine shape parameterization (see Section 1.1.3.5 for an example, and [85] for more details) naturally abides by the offline-online separability assumption, it often results in very limited deformation of the reference domain, or strong assumptions on the underlying shape.

Let $\Omega \subset \mathbb{R}^{d}, d=2,3$, be the reference domain. Let $\mathcal{M}$ be a parametric shape morphing function, that is,

$$
\mathcal{M}(\boldsymbol{x} ; \boldsymbol{\mu}): \mathbb{R}^{d} \rightarrow \mathbb{R}^{d}
$$

which maps the reference domain $\Omega$ into the deformed domain $\Omega(\boldsymbol{\mu})$ as $\Omega(\boldsymbol{\mu})=$ $\mathcal{M}(\Omega ; \boldsymbol{\mu})$, where $\boldsymbol{\mu} \in \mathcal{P}$ represents the vector of the geometrical parameters. This map will change accordingly to the chosen shape morphing technique. The case of Section 1.1.3.5 is representative of an affine map $\mathcal{M}(\cdot ; \boldsymbol{\mu})$. Instead, in the following we address more general (not necessarily affine) techniques such as FFD, RBF interpolation, and IDW interpolation.

From a practical point of view, we recommend the Python package called PyGeM Python Geometrical Morphing [79], which allows an easy integration with the majority of industrial CAD files and the most common mesh files.

\subsubsection{Free form deformation}

Free form deformation (FFD) is a widely used parameterization and morphing technique both in academia and in industry. 
For the original formulation see [94]. More recent works use FFD coupled with reduced basis methods for shape optimization and design of systems modeled by elliptic PDEs (see [63], [86], and [96]), in naval engineering for the optimization of the bulbous bow shape of cruise ships (in [37]), in the context of sailing boats in [65], and in automotive engineering in [91].

FFD can be used for both global and local deformations and it is completely independent of the geometry to morph. It acts through the displacement of a lattice of points, called FFD control points, constructed around the domain of interest. In particular it consists in three different steps, as depicted in Figure 1.3. First the physical domain $\Omega$ is mapped to $\hat{\Omega}$, the reference one, through the affine map $\boldsymbol{\psi}$. Then the lattice of control points is constructed, and the displacements of these points by the map $\hat{T}$ is what we call geometrical parameters $\boldsymbol{\mu}$. The deformation is propagated to the entire embedded body usually by using Bernstein polynomials. Finally through the inverse map $\boldsymbol{\psi}^{-1}$ we return back to the parametric physical space $\Omega(\boldsymbol{\mu})$.

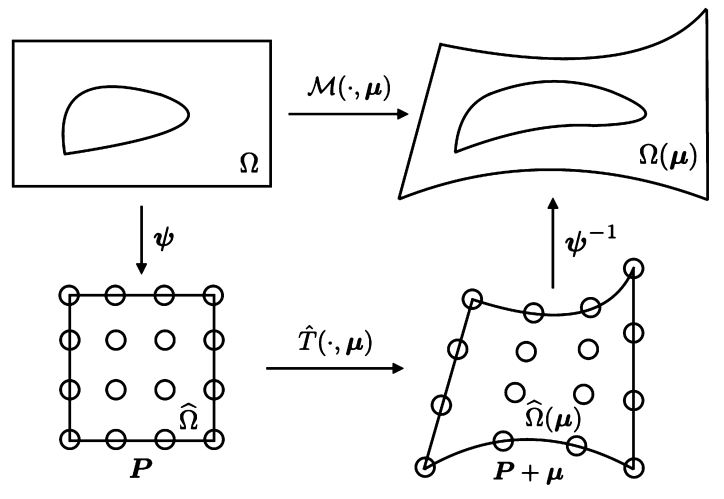

Figure 1.3: Scheme of the three maps composing the FFD map $\mathcal{M}$. In particular $\psi$ maps the physical space to the reference one, then $\hat{T}$ deforms the entire geometry according to the displacements of the lattice control points, and finally $\psi^{-1}$ maps back the reference domain to the physical one.

So, recalling equation (1.60), we have the explicit map $\mathcal{M}$ for the FFD, that is, the composition of the three maps presented, i.e.,

$$
\begin{aligned}
\mathcal{M}(\boldsymbol{x}, \boldsymbol{\mu}) & =\left(\boldsymbol{\psi}^{-1} \circ \widehat{T} \circ \boldsymbol{\psi}\right)(\boldsymbol{x}, \boldsymbol{\mu})= \\
& =\boldsymbol{\psi}^{-1}\left(\sum_{l=0}^{L} \sum_{m=0}^{M} \sum_{n=0}^{N} b_{l m n}(\boldsymbol{\psi}(\boldsymbol{x})) \boldsymbol{P}_{l m n}^{0}\left(\boldsymbol{\mu}_{l m n}\right)\right) \quad \forall \boldsymbol{x} \in \Omega,
\end{aligned}
$$

where $b_{l m n}$ are Bernstein polynomials of degree $l, m, n$ in each direction, respectively, and $\boldsymbol{P}_{l m n}^{0}\left(\boldsymbol{\mu}_{l m n}\right)=\boldsymbol{P}_{l m n}+\boldsymbol{\mu}_{l m n}$, with $\boldsymbol{P}_{l m n}$ representing the coordinates of the control point identified by the three indices $l, m, n$ in the lattice of FFD control points. In an offline-online fashion, for a given $\boldsymbol{x}$, terms $\left\{b_{l m n}(\boldsymbol{\psi}(\boldsymbol{x}))\right\}_{l, m, n}$ can be precomputed during the offline stage, resulting in an inexpensive linear combination of $\boldsymbol{x}$-dependent precomputed quantities and $\boldsymbol{\mu}$-dependent control points locations $\left\{\boldsymbol{P}_{l m n}^{0}\left(\boldsymbol{\mu}_{l m n}\right)\right\}_{l, m, n}$. The application of $\boldsymbol{\psi}^{-1}$ does not hinder such offline-online approach as $\boldsymbol{\psi}$ is affine. 
We can notice that the deformation does not depend on the topology of the object to be morphed, so this technique is very versatile and nonintrusive, especially for complex geometries or in industrial contexts (see, e. g., [90, 87]).

In the case where the deformation has to satisfy some constraints, like for example continuity constraints, it is possible to increase the number of control points. Often it is the case where at the interface between the undeformed portion of the geometry and the morphed area the continuity has to be prescribed for physical reasons.

As an example, in Figure 1.4 we present an FFD of a bulbous bow, where an STL file of a complete hull is morphed continuously by the displacement of only some control points.

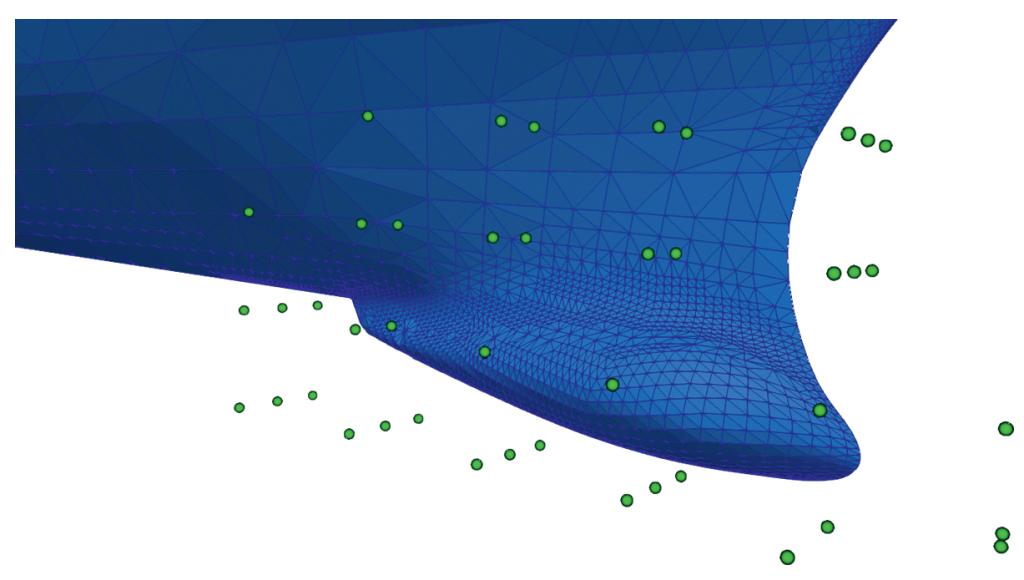

Figure 1.4: Bulbous bow deformation using FFD. In green are shown the FFD control points defining the morphing.

\subsubsection{Radial basis function interpolation}

Radial basis functions (RBFs) represent a powerful tool for nonlinear multivariate approximation, interpolation between nonconforming meshes ([40]), and shape parameterization due to their approximation properties [15].

An $\mathrm{RBF}$ is any smooth real-valued function $\widetilde{\varphi}: \mathbb{R}^{d} \rightarrow \mathbb{R}$ such that $\varphi: \mathbb{R}^{+} \rightarrow \mathbb{R}$ exists and $\widetilde{\varphi}(\boldsymbol{x})=\varphi(\|\boldsymbol{x}\|)$, where $\|\cdot\|$ indicates the Euclidean norm in $\mathbb{R}^{d}$. The most widespread RBFs are the following:

- Gaussian splines ([15]) defined as

$$
\varphi(\|\boldsymbol{x}\|)=e^{-\|\boldsymbol{x}\|^{2} / R}
$$

- thin plate splines ([42]) defined as

$$
\varphi(\|\boldsymbol{x}\|)=\left(\frac{\|\boldsymbol{x}\|}{R}\right)^{2} \ln \left(\frac{\|\boldsymbol{x}\|}{R}\right) ;
$$


- $\quad$ Beckert and Wendland $C^{2}$-basis ([11]) defined as

$$
\varphi(\|\boldsymbol{x}\|)=\left(1-\frac{\|\boldsymbol{x}\|}{R}\right)_{+}^{4}\left(4 \frac{\|\boldsymbol{x}\|}{R}+1\right) ;
$$

- multiquadratic biharmonic splines ([92]) defined as

$$
\varphi(\|\boldsymbol{x}\|)=\sqrt{\|\boldsymbol{x}\|^{2}+R^{2}} ;
$$

- $\quad$ inverted multiquadratic biharmonic splines ([15]) defined as

$$
\varphi(\|\boldsymbol{x}\|)=\frac{1}{\sqrt{\|\boldsymbol{x}\|^{2}+R^{2}}} ;
$$

where $R>0$ is a given radius and the subscript ${ }_{+}$indicates the positive part.

Following [75, 72], given $\mathcal{N}_{C}$ control points situated on the surface of the body to morph, we can generate a deformation by moving some of these points and imposing the new surface which interpolates them. The displacements of the control points represent the geometrical parameters $\boldsymbol{\mu}$.

We can now define the map $\mathcal{M}$ in equation (1.60) for the RBF interpolation technique, that is,

$$
\mathcal{M}(\boldsymbol{x} ; \boldsymbol{\mu})=q(\boldsymbol{x} ; \boldsymbol{\mu})+\sum_{i=1}^{\mathcal{N}_{C}} \gamma_{i}(\boldsymbol{\mu}) \varphi\left(\left\|\boldsymbol{x}-\boldsymbol{x}_{C_{i}}\right\|\right),
$$

where $q(\boldsymbol{x} ; \boldsymbol{\mu})$ is a polynomial term, generally of degree $1, \gamma_{i}(\boldsymbol{\mu})$ is the weight associated to the basis function $\varphi_{i},\left\{\boldsymbol{x}_{C_{i}}\right\}_{i=1}^{\mathcal{N}_{C}}$ are control points selected by the user (denoted by spherical green markers in Figure 1.5), and $\boldsymbol{x} \in \Omega$. We underline that in the threedimensional case (1.63) has $d \times \mathcal{N}_{C}+d+d^{2}$ unknowns, which are $d \times \mathcal{N}_{C}$ for $\gamma_{i}$ and $d+d^{2}$ for the polynomial term $q(\boldsymbol{x} ; \boldsymbol{\mu})=c(\boldsymbol{\mu})+\mathbf{Q}(\boldsymbol{\mu}) \boldsymbol{x}$. To this end we impose the interpolatory constraint

$$
\mathcal{M}\left(\boldsymbol{x}_{C_{i}} ; \boldsymbol{\mu}\right)=\boldsymbol{y}_{C_{i}}(\boldsymbol{\mu}) \quad \forall i \in\left\{1, \ldots, \mathcal{N}_{C}\right\}
$$

where $\boldsymbol{y}_{C_{i}}$ are the deformed control points obtained applying the displacement $\boldsymbol{\mu}$ to $\boldsymbol{x}_{C_{i}}$, in particular

$$
\begin{aligned}
\boldsymbol{x}_{C} & =\left[\boldsymbol{x}_{C_{1}}, \ldots, \boldsymbol{x}_{C_{\mathcal{N}_{C}}}\right] \in \mathbb{R}^{\mathcal{N}_{C} \times d}, \\
\boldsymbol{y}_{C}(\boldsymbol{\mu}) & =\left[\boldsymbol{y}_{C_{1}}(\boldsymbol{\mu}), \ldots, \boldsymbol{y}_{C_{\mathcal{N}_{C}}}(\boldsymbol{\mu})\right] \in \mathbb{R}^{\mathcal{N}_{C} \times d} .
\end{aligned}
$$

For the remaining $d+d^{2}$ unknowns, due to the presence of the polynomial term, we complete the system with additional constraints that represent the conservation of the total force and momentum $[15,75]$ as follows:

$$
\sum_{i=1}^{\mathcal{N}_{C}} \gamma_{i}(\boldsymbol{\mu})=0
$$





Figure 1.5: Two different views of the same deformed carotid artery model using the RBF interpolation technique. The green dots indicate the RBF control points that define the morphing. The black small points highlight the original undeformed geometry. The occlusion of the two branches is achieved through a displacement along the normal direction with respect to the carotid surface of the control points after the bifurcation.

$$
\sum_{i=1}^{\mathcal{N}_{C}} \gamma_{i}(\boldsymbol{\mu})\left[\boldsymbol{x}_{C_{i}}\right]_{1}=0, \ldots \sum_{i=1}^{\mathcal{N}_{C}} \gamma_{i}(\boldsymbol{\mu})\left[\boldsymbol{x}_{C_{i}}\right]_{d}=0,
$$

where the notation $[\boldsymbol{x}]_{d}$ denotes the $d$-th component of the vector $\boldsymbol{x}$.

Following an offline-online strategy, for a given $\boldsymbol{x}$, evaluation of $\varphi\left(\left\|\boldsymbol{x}-\boldsymbol{x}_{C_{i}}\right\|\right), i=$ $1, \ldots, \mathcal{N}_{C}$, can be precomputed in the offline stage. Further online effort is only required for (i) given $\boldsymbol{\mu}$, solve a $d \times \mathcal{N}_{C}+d+d^{2}$ linear system, and (ii) given $\boldsymbol{\mu}$ and $\boldsymbol{x}$, perform linear combinations and the matrix vector product in (1.63) employing either precomputed quantities or coefficients from (i).

\subsubsection{Inverse distance weighting interpolation}

The Inverse distance weighting (IDW) interpolation method has been proposed in [95] to deal with interpolation of scattered data. We follow [108, 49, 9] for its presentation and the application of IDW to shape parameterization.

As in the previous section, let $\left\{\boldsymbol{x}_{C_{k}}\right\}_{k=1}^{\mathcal{N}_{c}} \subset \mathbb{R}^{d}$ be a set of control points. The IDW interpolant $\Pi_{\mathrm{IDW}}(f)$ of a scalar function $f: \mathbb{R}^{d} \rightarrow \mathbb{R}$ is defined as

$$
\Pi_{\mathrm{IDW}}(f)(\boldsymbol{x})=\sum_{k=1}^{\mathcal{N}_{c}} w_{k}(\boldsymbol{x}) f\left(\boldsymbol{x}_{C_{k}}\right), \quad \boldsymbol{x} \in \Omega,
$$


where the weight functions $w_{k}: \Omega \rightarrow \mathbb{R}$, for $k=1, \ldots, \mathcal{N}_{c}$ are given by

$$
w_{k}(\boldsymbol{x})= \begin{cases}\frac{\left\|\boldsymbol{x}-\boldsymbol{x}_{C_{k}}\right\|^{-s}}{\sum_{j=1}^{\mathcal{N}_{c}}\left\|\boldsymbol{x}-\boldsymbol{x}_{C_{j}}\right\|^{-s}} & \text { if } \boldsymbol{x} \neq \boldsymbol{x}_{C_{k}}, \\ 1 & \text { if } \boldsymbol{x}=\boldsymbol{x}_{C_{k}}, \\ 0 & \text { otherwise }\end{cases}
$$

where $s$ is a positive integer, modeling the assumption that the influence of the $k$-th control point $\boldsymbol{x}_{C_{k}}$ on $\boldsymbol{x}$ diminishes with rate $-s$ as the distance between $\boldsymbol{x}$ and $\boldsymbol{x}_{C_{k}}$ increases. IDW interpolation trivially extends to vector functions $\boldsymbol{f}: \mathbb{R}^{d} \rightarrow \mathbb{R}^{d}$ by application to each component $f_{1}, \ldots, f_{d}$, where the weight functions $w_{k}: \Omega \rightarrow \mathbb{R}$ do not depend on the specific component.

In the case of IDW shape parameterization, for any given $\boldsymbol{\mu}$, the deformed position of the control points $\left\{\boldsymbol{x}_{C_{k}}\right\}_{k=1}^{\mathcal{N}_{c}}$ is supposed to be known, and equal to $\boldsymbol{y}_{C_{k}}(\boldsymbol{\mu}):=\boldsymbol{f}\left(\boldsymbol{x}_{C_{k}}\right)$ for $k=1, \ldots, \mathcal{N}_{c}$. We remark that the analytic expression of $\boldsymbol{f}$ is not known, but only its action through $\left\{\boldsymbol{x}_{C_{k}}\right\}_{k=1}^{\mathcal{N}_{c}}$. This is indeed the minimum requirement to properly define (1.69). The deformation map is therefore

$$
\mathcal{M}(\boldsymbol{x} ; \boldsymbol{\mu})=\sum_{k=1}^{\mathcal{N}_{c}} w_{k}(\boldsymbol{x}) \boldsymbol{y}_{C_{k}}(\boldsymbol{\mu}) \quad \forall \boldsymbol{x} \in \Omega .
$$

In an offline-online separation effort, efficient deformation can be obtained by noting that the $\boldsymbol{\mu}$-dependent part is decoupled from the $\boldsymbol{x}$-dependent weight function $w_{k}(\boldsymbol{x})$. Thus, for any $\boldsymbol{x}$, weight terms can be precomputed once and for all and stored. The online cost of the evaluation of $\mathcal{M}(\boldsymbol{x} ; \boldsymbol{\mu})$ thus requires an inexpensive linear combination of $\boldsymbol{x}$-dependent precomputed quantities and $\boldsymbol{\mu}$-dependent control point locations. We remark that, in contrast, the RBF approach (even though still based on interpolation) required a further solution of linear system of size $d \times \mathcal{N}_{C}+d+d^{2}$.

Application in the context of fluid-structure interaction problems between a wing (structure) and surrounding air (fluid) is shown in Figure 1.6. The IDW deformation of

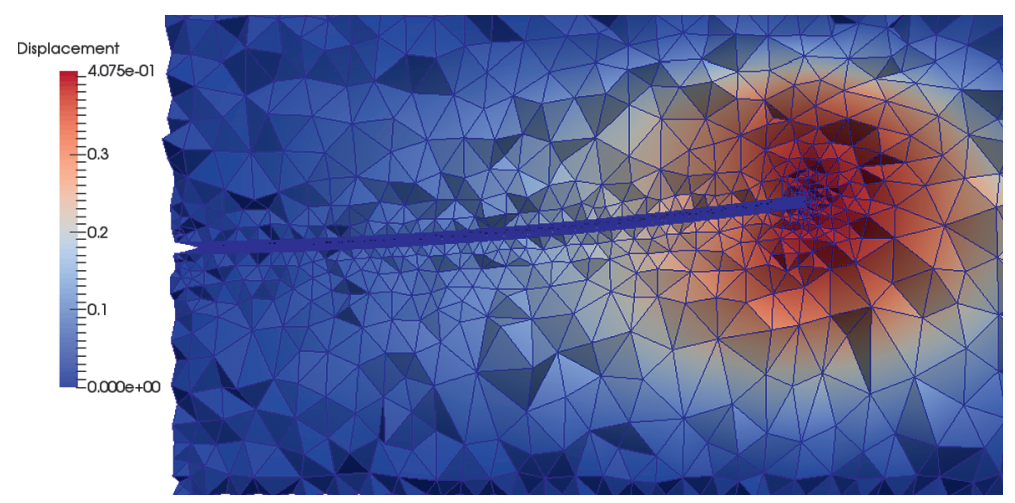

Figure 1.6: Deformation of the fluid mesh of a fluid-structure interaction problem by IDW. 
the fluid mesh resulting from a vertical displacement of the tip of the wing is depicted; the structural mesh is omitted from the picture. We refer to [9] for more details.

\subsection{Beyond affinity assumptions: parametric interpolation}

We describe here several options to deal with cases when an exact affine decomposition of the discretized differential operators, right-hand sides, or outputs of interest does not exist. The section begins with a brief overview concerning the description of general nonaffine problems in Section 1.3.1 and later we describe the so-called EIM family of algorithms. This methodology becomes particularly useful to obtain an efficient offline-online splitting also in cases with nonlinearities and nonaffine parameterization. We provide a full description of the different alternatives, starting from its standard continuous version (EIM), and presenting also its discrete (DEIM) and matrix (M-DEIM) variants. The methodologies are tested for both nonaffine and nonlinear problems. In Section 1.3.2 we explain in detail the basics of the EIM. In Section 1.3.3 we introduce the discrete variant of the EIM at both matrix and vector level and we mention further options to obtain an approximate affine expansion. In Section 1.3.5 we present two examples using the EIM (Section 1.3.5.1) and the M-DEIM algorithm to deal with both nonaffinity and nonlinearity (Section 1.3.5.2).

\subsubsection{Nonaffine problems}

As already discussed in Section 1.1.3.4, the existence of an affine decomposition of the linear and bilinear forms of the considered problem is crucial in order to obtain a computationally efficient framework (see (1.26)-(1.28)).

This assumption fails to be true in several situations. Such situations occur for example in case of problems with nonaffine parametric dependency, in cases with nonlinear differential operators, and in cases dealing with the nonaffine geometrical parameterizations introduced in Section 1.2.

In fact, in these situations, the differential operators, the right-hand sides, or the outputs of interest cannot be directly written using an exact affine decomposition and we have therefore to rely on an approximate affine decomposition. The EIM is one of the key instruments to recover an approximate affine decomposition.

The EIM is a general tool for the approximation of parameterized or nonlinear functions by a sum of affine terms. In the following expression we report an example for a generic parameterized function $f$ :

$$
f(\boldsymbol{x} ; \boldsymbol{\mu}) \approx \sum_{q=1}^{Q} c_{q}(\boldsymbol{\mu}) h_{q}(\boldsymbol{x}) .
$$


The EIM has been firstly proposed in [10] to deal with nonaffine problems in the context of reduced basis methods and later applied to ROM in [53]. In [69] it has been extended to a general context, and a slightly different variant of EIM, DEIM, has been firstly proposed in $[22,23]$. For more details on the a posteriori error analysis the interested reader may see $[53,44,24]$ while for an extension to $h p$-adaptive EIM we refer to [45]. A generalization of the EIM family of algorithms has been proposed in $[68,24,67]$ while a nonintrusive EIM technique is presented in [21] and an extension with special focus on high-dimensional parameter spaces is given in [56].

\subsubsection{The empirical interpolation method}

The EIM is a general method to approximate a parameterized function $f(\boldsymbol{x} ; \boldsymbol{\mu}): \Omega \times$ $\mathcal{P}_{\text {EIM }} \rightarrow \mathbb{R}$ by a linear combination of $Q$ precomputed basis functions in the case where each function $f_{\mu}:=(\cdot ; \boldsymbol{\mu})$ belongs to some Banach space $\mathcal{X}_{\Omega}$. In what follows $\boldsymbol{\mu} \in \mathcal{P}_{\text {EIM }}$ is the parameter vector and $\mathcal{P}_{\text {EIM }}$ is the parameter space. The EIM approximation is based on an interpolation operator $\mathrm{I}_{Q}$ that interpolates the given function $f_{\mu}$ in a set of interpolation points $\left\{\boldsymbol{x}_{i}\right\}_{i=1}^{Q} \in \Omega$. The interpolant function is constructed as a linear combination of hierarchically chosen basis functions $\left\{h_{i}\right\}_{i=1}^{Q} \in \mathbb{V}_{\text {EIM }}$, where $\mathbb{V}_{\text {EIM }}$ is an approximation of the function space $\mathcal{U}$ that contains $f$, i. e., $\mathbb{V}_{\text {EIM }} \subseteq \mathcal{U}$. On the contrary to other interpolation methods, that usually work with generic and multipurpose basis functions such as polynomial functions, the EIM works with problem-specific basis functions with global support and selected hierarchically. The interpolant function can be then expressed by

$$
\mathrm{I}_{Q}\left[f_{\mu}\right](\boldsymbol{x})=\sum_{q=1}^{Q} c_{q}(\boldsymbol{\mu}) h_{q}(\boldsymbol{x}), \quad \boldsymbol{x} \in \Omega, \quad \boldsymbol{\mu} \in \mathcal{P}_{\mathrm{EIM}},
$$

where $c_{q}$ are parameter-dependent coefficients. Once the basis functions $h_{q}(\boldsymbol{x})$ are set, the problem of finding the coefficients $c_{q}(\boldsymbol{\mu})$ is solved imposing the interpolation condition, i. e.,

$$
\mathrm{I}_{Q}\left[f_{\mu}\right]\left(\boldsymbol{x}_{q}\right)=\sum_{q=1}^{Q} c_{q}(\boldsymbol{\mu}) h_{q}\left(\boldsymbol{x}_{q}\right)=f_{\mu}\left(\boldsymbol{x}_{q}\right), \quad q=1, \ldots, Q
$$

The above problem can be recast in matrix form as $\boldsymbol{T} \boldsymbol{c}_{\mu}=\boldsymbol{f}_{\mu}$ with

$$
(\boldsymbol{T})_{i j}=h_{j}\left(\boldsymbol{x}_{i}\right), \quad\left(\boldsymbol{c}_{\mu}\right)_{j}=c_{j}(\boldsymbol{\mu}), \quad(\boldsymbol{f}(\boldsymbol{\mu}))_{j}=f\left(\boldsymbol{x}_{i} ; \boldsymbol{\mu}\right), \quad i, j=1, \ldots, Q
$$

This problem can be easily solved given the fact that the basis functions $h_{q}(\boldsymbol{x})$ and the interpolation points $\boldsymbol{x}_{q}$ are known and that the matrix $\boldsymbol{T}$ is invertible.

The selection of the basis functions $\left\{h_{q}\right\}_{q=1}^{Q}$ and of the interpolation points $\left\{\boldsymbol{x}_{q}\right\}_{q=1}^{Q}$, which are defined by a linear combination of selected function realizations $\left\{f_{\mu_{i}}\right\}_{i=1}^{Q}$, 
is done following a greedy approach similar to the one presented in Section 1.1.3.2 (Algorithm 1.2). The procedure provides also a set of sample points $\left\{\boldsymbol{\mu}_{q}\right\}_{q=1}^{Q}$ that are required for the construction of the basis functions.

Since the basis functions are defined as linear combinations of the function realizations inside the parameter space, in order to approximate the function $f$ with a relatively small number of basis functions $h_{q}$, the manifold

$$
\mathcal{M}_{\text {EIM }}=\left\{f(\boldsymbol{x} ; \boldsymbol{\mu}) \mid \boldsymbol{\mu} \in \mathcal{P}_{\text {EIM }}\right\}
$$

must have a small Kolmogorov $N$-width [61].

Once a proper norm on $\Omega$ has been defined, where we consider $L^{p}(\Omega)$-norms for $1 \leq p \leq \infty$, the procedure starts with the selection of the first parameter sample, which is computed as

$$
\boldsymbol{\mu}_{1}=\underset{\boldsymbol{\mu} \in \mathcal{P}_{\mathrm{EIM}}}{\arg \sup }\left\|f_{\mu}(\boldsymbol{x})\right\|_{L^{p}(\Omega)},
$$

while the first interpolation point is computed as

$$
\boldsymbol{x}_{1}=\underset{\boldsymbol{x} \in \Omega}{\arg \sup }\left|f_{\mu_{1}}(\boldsymbol{x})\right|
$$

The first basis function and the interpolation operator at this stage are then defined as

$$
h_{1}(x)=\frac{f_{\mu_{1}}(\boldsymbol{x})}{f_{\mu_{1}}\left(\boldsymbol{x}_{1}\right)}, \quad \mathrm{I}_{1}\left[f_{\mu}\right](\boldsymbol{x})=f\left(\boldsymbol{x}_{1} ; \boldsymbol{\mu}\right) h_{1}(\boldsymbol{x}) .
$$

At the subsequent steps, the next basis function is selected as the one that is the worse approximated by the current interpolation operator and using a similar concept the interpolation point, often referred as magic point, is the one where the interpolation error is maximized. In mathematical terms, at the step $k$, the sample point is selected as the one that maximizes the error between the function $f$ and the interpolation operator computed at the previous step $\mathrm{I}_{k-1}[f]$ :

$$
\boldsymbol{\mu}_{k}=\underset{\boldsymbol{\mu} \in \mathcal{P}_{\mathrm{EIM}}}{\arg \sup }\left\|f_{\mu}(\boldsymbol{x})-\mathrm{I}_{k-1}\left[f_{\mu}\right](\boldsymbol{x})\right\|_{L^{p}(\Omega)} .
$$

Once the sample point has been determined, the interpolation point is selected, in a similar fashion, as the point inside the domain that maximizes the error between the function $f$ and the interpolation operator:

$$
\boldsymbol{x}_{k}=\underset{\boldsymbol{x} \in \Omega}{\arg \sup }\left|f_{\mu_{k}}(\boldsymbol{x})-\mathrm{I}_{k-1}\left[f_{\mu_{k}}\right](\boldsymbol{x})\right| .
$$

The next basis function is defined similarly to the first one with

$$
h_{k}(\boldsymbol{x})=\frac{f_{\mu_{k}}(\boldsymbol{x})-\mathrm{I}_{k-1}\left[f_{\mu_{k}}\right](\boldsymbol{x})}{f_{\mu_{k}}\left(\boldsymbol{x}_{k}\right)-\mathrm{I}_{k-1}\left[f_{\mu_{k}}\right]\left(\boldsymbol{x}_{k} ;\right)} \text {. }
$$




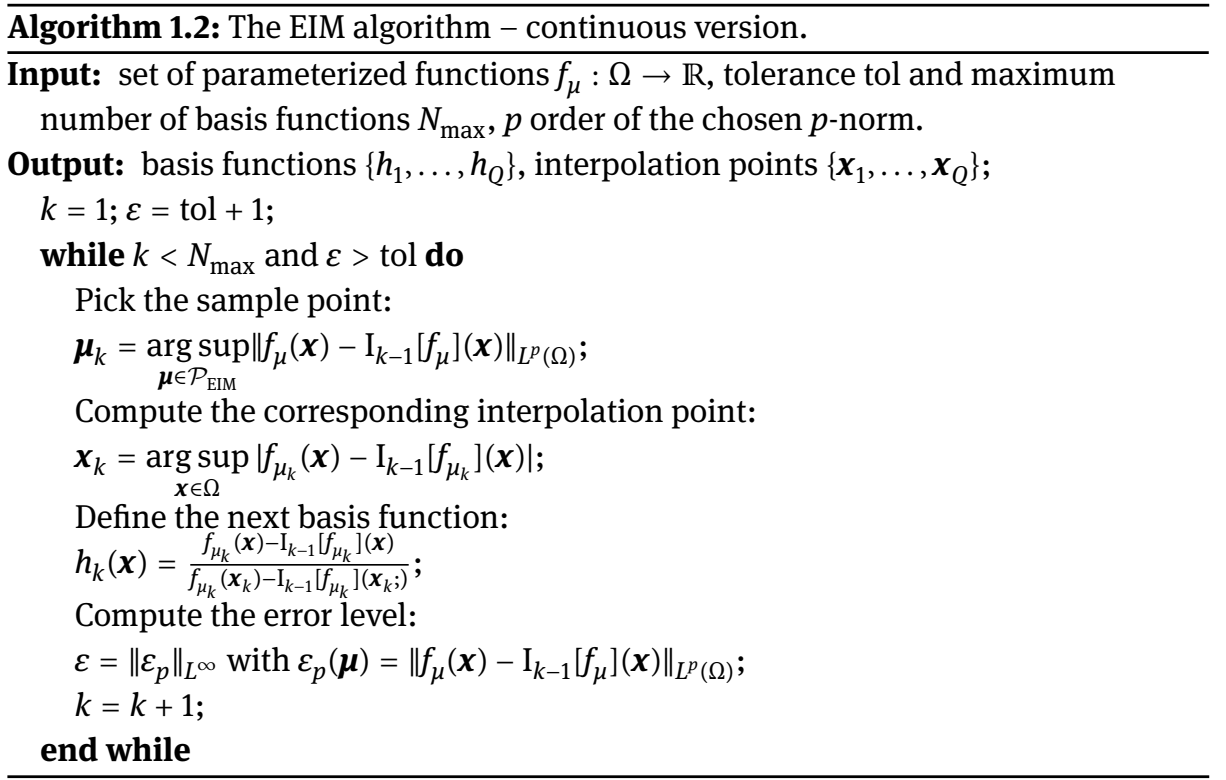

The procedure is repeated until a certain tolerance tol is reached or a maximum number of terms $N_{\max }$ are computed (Algorithm 1.2). We remark that by construction the basis functions $\left\{h_{1}, \ldots, h_{Q}\right\}$ and the functions $\left\{f_{\mu_{1}}, \ldots, f_{\mu_{Q}}\right\}$ span the same space $\mathbb{V}_{\text {EIM }}$ :

$$
\mathbb{V}_{\text {EIM }}=\operatorname{span}\left\{h_{1}, \ldots, h_{Q}\right\}=\operatorname{span}\left\{f_{\mu_{1}}, \ldots, f_{\mu_{Q}}\right\}
$$

However, the former are preferred for the following reasons (for more details and for the mathematical proofs we refer to [10]):

- they are linearly independent,

- $\quad h_{i}\left(\boldsymbol{x}_{i}\right)=1$ for $1 \leq i \leq Q$ and $h_{i}\left(\boldsymbol{x}_{j}\right)=0$ for $1 \leq i \leq j \leq Q$,

- they make the interpolation matrix $\boldsymbol{T}$ of equation (1.74) to be lower triangular and with diagonal elements equal to unity and therefore the matrix is invertible.

The third point implies that the interpolation problem is well-posed.

\subsubsection{Error analysis}

Dealing with interpolation procedures, the error analysis usually involves a Lebesgue constant. In particular, in the case one is using the $L^{\infty}(\Omega)$-norm the error analysis involves the computation of the Lebesgue constant $\Lambda_{q}=\sup _{\boldsymbol{x} \in \Omega} \sum_{i=1}^{q}\left|L_{i}(\boldsymbol{x})\right|$ being $L_{i} \epsilon$ $\mathbb{V}_{\text {EIM }}$ a Lagrange function that satisfies $L_{i}\left(x_{j}\right)=\delta_{i j}$. It can be proved that the interpola- 
tion error is bounded by the following expression [10]:

$$
\left\|f_{\mu}-\mathrm{I}_{\mathrm{q}}\left[f_{\mu}\right]\right\|_{L^{\infty}(\Omega)} \leq\left(1+\Lambda_{q}\right) \inf _{v_{q} \in \mathbb{V}_{\mathrm{EIM}}}\left\|f_{\mu}-v_{q}\right\|_{L^{\infty}(\Omega)} .
$$

An upper bound for the Lebesgue constant, which in practice has been demonstrated to be very conservative [10], can be computed as

$$
\Lambda_{q} \leq 2^{q}-1
$$

For more details concerning the estimates of the interpolation error we refer to $[10,69]$.

\subsubsection{Practical implementation of the algorithm}

Practically, finding the maximum of Algorithm 1.2 is usually not feasible and therefore the continuous version must be transformed into a computable one.

This is done selecting a finite-dimensional set of training points in the parameter space $\left\{\boldsymbol{\mu}_{i}\right\}_{i=1}^{N} \in \mathcal{P}_{\text {EIM }}^{\text {train }} \subset \mathcal{P}_{\text {EIM }}$ and in the physical domain $\left\{\boldsymbol{x}_{i}\right\}_{i=1}^{M} \in \Omega_{h} \subset \Omega$. For this reason we introduce the vector $\boldsymbol{f}: \Omega_{h} \times \mathcal{P}_{\mathrm{EIM}}^{\text {train }} \rightarrow \mathbb{R}^{M}$ which consists of a discrete representation of the function $f$ :

$$
\left(\boldsymbol{f}_{\mu}\right)_{i}=f_{\mu}\left(\boldsymbol{x}_{i}\right), \quad i=1, \ldots, M
$$

We also define the matrix $\boldsymbol{H}_{Q} \in \mathbb{R}^{M \times Q}$, which is defined by the discrete basis functions $H_{Q}=\left[\boldsymbol{h}_{1}, \ldots, \boldsymbol{h}_{Q}\right]$ and the interpolation index vector $\boldsymbol{i}_{Q}=\left(i_{1}, \ldots, i_{Q}\right)$. The discrete interpolation operator of order $Q$ for the vector function $\boldsymbol{f}$ is then defined by

$$
\mathrm{I}_{Q}\left[\boldsymbol{f}_{\boldsymbol{\mu}}\right]=\boldsymbol{H}_{Q} \boldsymbol{a}_{\boldsymbol{f}_{\boldsymbol{\mu}}}
$$

where the coefficients $\boldsymbol{a}_{f_{\mu}}$ are defined such that $\boldsymbol{T} \boldsymbol{a}_{\boldsymbol{f}_{\mu}}=\boldsymbol{f}_{\boldsymbol{\mu}}$, where

$$
\boldsymbol{T}_{k q}=\left(\boldsymbol{H}_{Q}\right)_{i_{k} q}, \quad k, q=1, \ldots, Q .
$$

The implementation of the algorithm is similar to the continuous version and is reported in Algorithm 1.3. In the algorithm we use the notation $\boldsymbol{F}_{: j}$ to denote the $j$-th column of the matrix $\boldsymbol{F}$, where $\boldsymbol{F} \in \mathbb{R}^{M \times N}$ is a matrix containing vector representations of the function $\boldsymbol{f}$ :

$$
(\boldsymbol{F})_{i j}=f\left(\boldsymbol{x}_{i} ; \boldsymbol{\mu}_{j}\right)
$$

Once the basis and the interpolation indices are defined, during the online stage it is required to make a pointwise evaluation of the $f$ function in the points defined by the interpolation indices. 


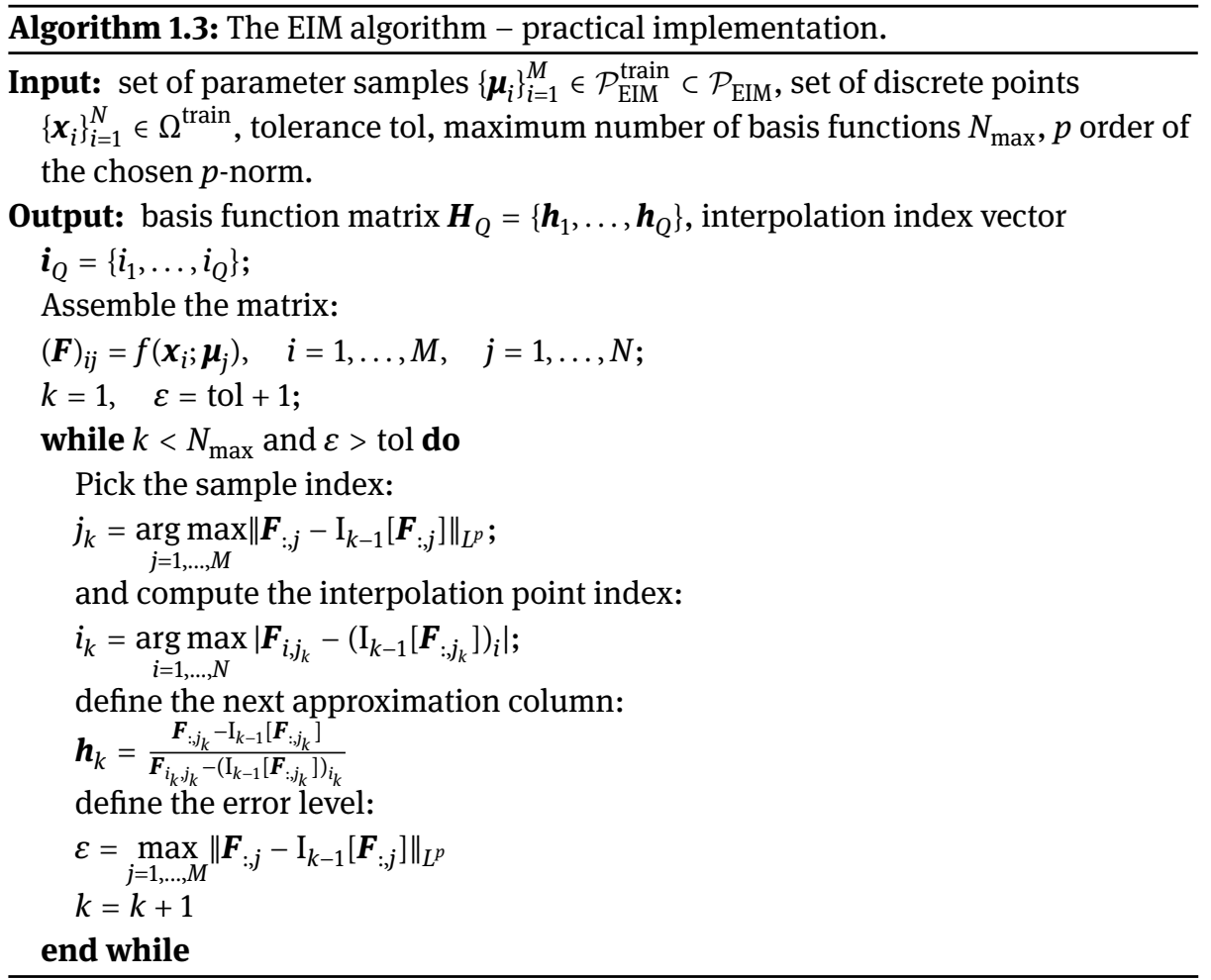

\subsubsection{The discrete empirical interpolation method}

A computable version of EIM is the so-called DEIM, introduced in [23]. We provide here an example as a special case of EIM where appropriate basis functions are already available [69]. In our example here DEIM basis functions are computed relying on a POD procedure which is performed on a set of discrete snapshots of the parameterized function $\left\{\boldsymbol{f}_{i}\right\}_{i=1}^{M}$. Each snapshot $\boldsymbol{f}_{i}$ is already considered in discrete form in a prescribed set of points $\left\{\boldsymbol{x}_{i}\right\}_{i=1}^{N_{h}}$. The procedure, which is described in detail in Algorithm 1.4, can be summarized into the following steps:

1. Construct the DEIM basis functions using a POD procedure on a set of previously computed snapshots:

$$
\boldsymbol{H}_{M}=\left[\boldsymbol{h}_{1}, \ldots, \boldsymbol{h}_{M}\right]=\operatorname{POD}\left(\boldsymbol{f}\left(\boldsymbol{\mu}_{1}, \ldots, \boldsymbol{\mu}_{M}\right)\right) .
$$

2. Given a prescribed tolerance tol, determine the indices $\boldsymbol{i}_{Q}$ and truncate the dimension of the POD space using an iterative greedy approach (Algorithm 1.4). 


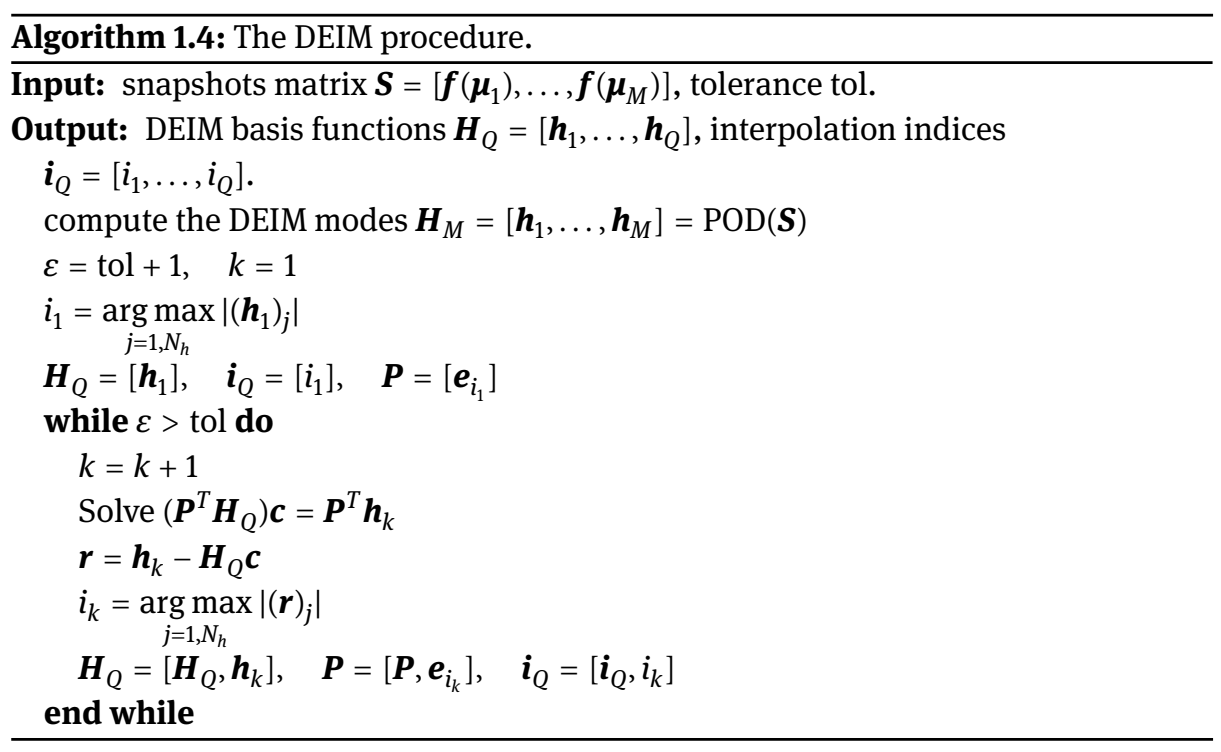

In Algorithm 1.4, with the term $\boldsymbol{e}_{i_{k}}$, we identify a vector of dimension $N_{h}$ where the only nonnull element is equal to 1 and is located at the index $i_{k}$ :

$$
\left(\boldsymbol{e}_{i_{k}}\right)_{j}=1 \text { for } j=i_{k}, \quad\left(\boldsymbol{e}_{i_{k}}\right)_{j}=0 \text { for } j \neq i_{k} .
$$

During the online stage, when a new value of the parameter $\boldsymbol{\mu}$ needs to be tested, it is required to compute the function $\boldsymbol{f}(\boldsymbol{\mu})$ only in the location identified by the indices $\boldsymbol{i}_{Q}$. Therefore, the nonlinear function needs to be evaluated only in a relatively small number of points which is usually much smaller with respect to the total number of degrees of freedom used to discretize the domain.

\subsubsection{Further options}

Apart from the EIM and the DEIM algorithm, further options are available. We mention here the matrix version of the DEIM algorithm (M-DEIM) [14], which extends the DEIM also to the case of parameterized or nonlinear matrices, the generalized EIM (GEIM) [68], and the gappy POD [16, 20].

The M-DEIM is used to perform MOR on discretized differential operators characterized by nonlinearity or nonaffinity with respect to the parameter vector $\boldsymbol{\mu}$. The algorithm is similar to the one in Algorithm 1.4 with the only difference that a vectorized version of the matrices is used to describe snapshots and POD modes. In Section 1.3.5 we will provide an example dealing with both issues. 
The gappy POD generalizes the interpolation condition to the case where the number of basis functions is smaller than the number of interpolation indices, i. e., $\operatorname{card}\left(\boldsymbol{H}_{Q}\right)<\operatorname{card}\left(\boldsymbol{i}_{Q}\right)$. In this case the interpolation condition is substituted by a leastsquares regression.

The GEIM replaces the EIM requirement of a pointwise interpolation condition by the following statement:

$$
\sigma_{j}\left(\mathrm{I}_{Q}(f(\boldsymbol{\mu}))\right)=\sigma_{j}(f(\boldsymbol{\mu})), \quad j=1, \ldots, Q,
$$

where $\sigma_{j}$ are a set of "well-chosen" linear functionals. For more details and for convergence analysis of the present method we refer to [67].

\subsubsection{Some examples}

In the previous sections we have presented the EIM family of algorithms and we have illustrated how it is possible to recover an approximate affine expansion of the discretized differential operators. In this section we show in more detail two examples on the practical application of the EIM and the M-DEIM algorithm.

\subsubsection{A heat transfer problem with a parameterized nonaffine dependency forcing term}

In this example we illustrate the application of the computable version of the EIM on a steady-state heat conduction problem in a two-dimensional square domain $\Omega=$ $[-1,1]^{2}$ with a parameterized forcing term $g(\boldsymbol{\mu})$ and homogeneous Dirichlet boundary conditions on the boundary $\partial \Omega$. The problem is described by the following equation:

$$
\left\{\begin{array}{l}
-\alpha_{t} \Delta \theta=g(\boldsymbol{\mu}), \quad \text { in } \Omega, \\
\theta=0, \quad \text { on } \partial \Omega,
\end{array}\right.
$$

where $\theta$ is the temperature field, $\alpha_{t}$ is the thermal conductivity coefficient, and $g(\boldsymbol{\mu})$ is the parameterized forcing term which is described by the following expression:

$$
g(\boldsymbol{x} ; \boldsymbol{\mu})=e^{-2\left(x_{1}-\mu_{1}\right)^{2}-2\left(x_{2}-\mu_{2}\right)^{2}},
$$

where $\mu_{1}$ and $\mu_{2}$ are the first and second components of the parameter vector and $x_{1}$ and $x_{2}$ are the horizontal and vertical coordinates, respectively. Let $V$ be a Hilbert space. The weak formulation of the problem can be written as follows: Find $\theta \in V$ such that

$$
a(\theta(\boldsymbol{\mu}), v ; \boldsymbol{\mu})=f(v ; \boldsymbol{\mu}), \quad \forall v \in V,
$$


where the parameterized bilinear and linear forms are expressed by

$$
a(\theta, v ; \boldsymbol{\mu})=\int_{\Omega} \nabla \theta \cdot \nabla v d \boldsymbol{x}, \quad f(v ; \boldsymbol{\mu})=\int_{\Omega} g(\boldsymbol{x} ; \boldsymbol{\mu}) v d \boldsymbol{x} .
$$

In the above expressions, the bilinear form $a(\cdot, ; \boldsymbol{\mu}): V \times V \rightarrow \mathbb{R}$ is trivially affine while for the linear form $f(\cdot ; \boldsymbol{\mu}): V \rightarrow \mathbb{R}$ we have to rely on an approximate affine expansion using the EIM. The problem is discretized using triangular linear finite elements according to the mesh reported on the left side of Figure 1.9.

In the present case it is not possible to write an exact affine decomposition of the linear form $f$; we rely therefore on the computable version of the EIM of Algorithm 1.3 in order to recover an approximate affine expansion.

The function $g(\boldsymbol{x} ; \boldsymbol{\mu})$ is parameterized with the parameter vector $\boldsymbol{\mu}=\left(\mu_{1}, \mu_{2}\right) \epsilon$ $\mathcal{P}_{\text {EIM }}=[-1,1]^{2}$, which describes the position of the center of the Gaussian function. The conductivity coefficient $\alpha_{t}$ is fixed constant and equal to 1 . The testing set for the implementation of the algorithm $\left\{\boldsymbol{\mu}_{i}\right\}_{i=N_{\text {train }}} \in \mathcal{P}_{\text {train }}$ is defined using $N_{\text {train }}=100$ and a uniform probability distribution. The set of points $\left\{\boldsymbol{x}_{i}\right\}_{i=1}^{N_{h}} \in \Omega$ that is used for the identification of the magic points is chosen to be coincident with the nodes of the finite element grid reported in Figure 1.7. In Figure 1.8 we report the first four EIM basis functions for the nonlinear function $g$ and the location of the magic points identified by the EIM algorithm. In Figure 1.9 we report the convergence analysis of the EIM algorithm for the nonlinear function $g$ changing the number of EIM basis functions (left plot) and the convergence analysis of the ROM changing the number of reduced basis functions (right plot).
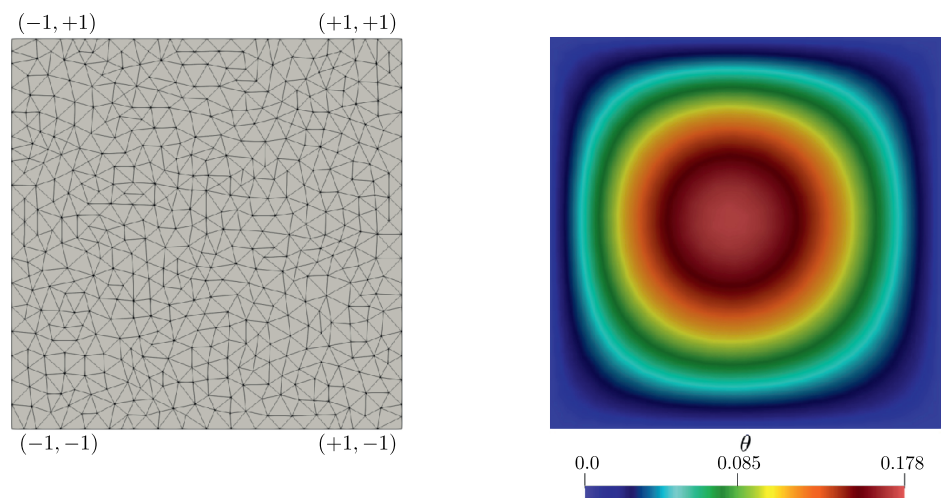

Figure 1.7: Discretized domain into which the parameterized problem is solved (left image), together with an example of the value assumed by the temperature field for one particular sample point inside the parameter space (right image). 


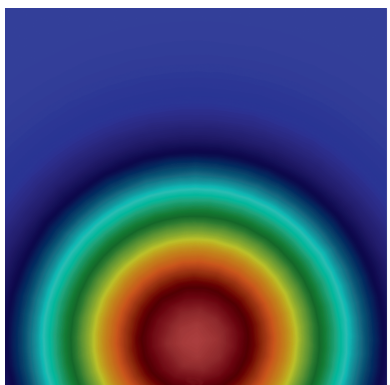

$0.00 e+00$

0.5

$1.00 e+00$



$0.00 e+00$

0.5

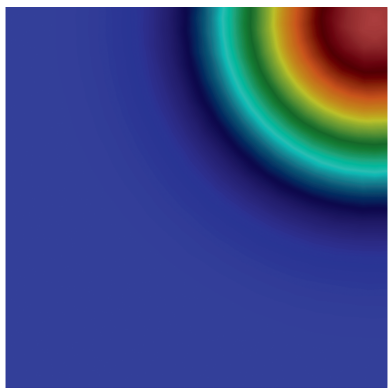

$0.00 e+00$

0.5

$1.00 \mathrm{e}+00$

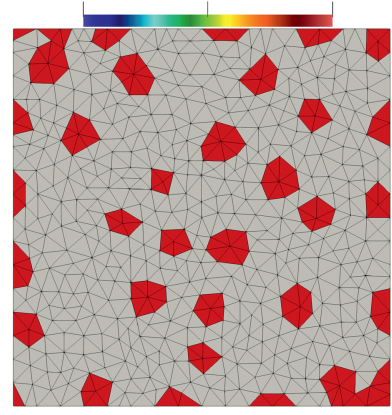

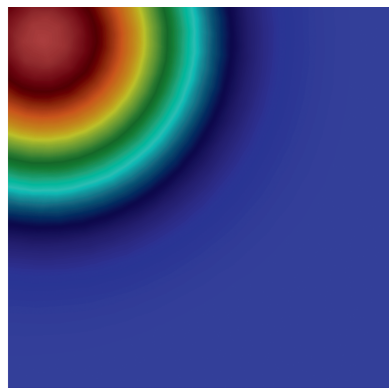

$0.00 e+00$

0.5

$1.00 e+00$

Figure 1.8: Plot of the first four modes identified by the EIM algorithm (first row and left image in the second row) and the location of the first 35 indices $i_{Q}$. The magic points are identified by the red elements in the right picture on the second row.
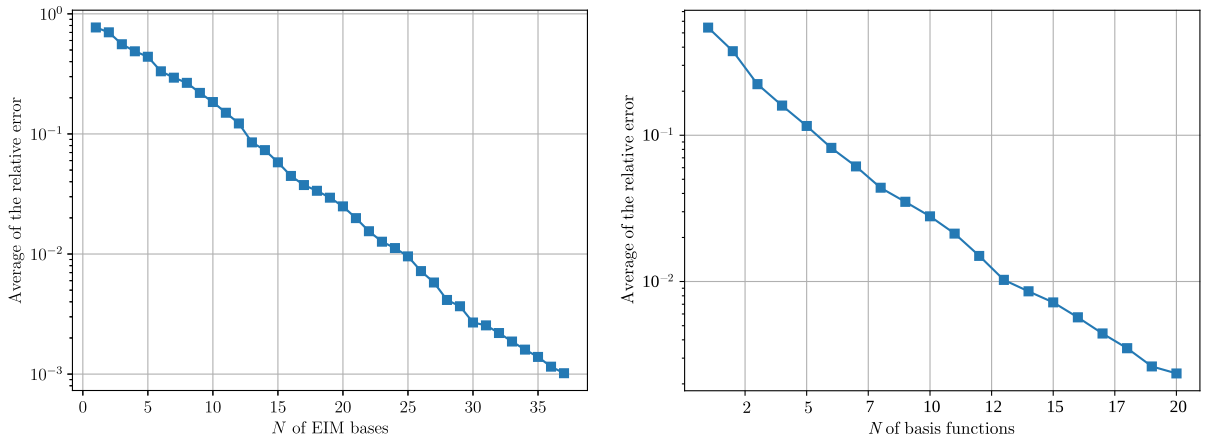

Figure 1.9: Convergence analysis of the numerical example. In the left plot we can see the average value of the $L^{2}$ relative error between the exact function $g$ and its EIM approximation. On the right plot we report the average value of the $L^{2}$ relative error between the FOM temperature field and the ROM temperature field. The plot is for different numbers of basis functions used to approximate the temperature field and keeping constant the number of basis functions used to approximate the forcing term $(N=11)$. 


\subsubsection{An example in the context of reduced-order models with nonlinearity and nonaffine parametric dependency}

In this second illustrative example we show the application of the DEIM algorithm to the stationary parameterized Navier-Stokes equations. In the present case we have both nonlinearity and nonaffinity with respect to the input parameters. Both nonlinearity and nonaffinity have been tackled using the matrix version of the DEIM. The computational domain is given by the unit square $\Omega=[0,1]^{2}$ and the physical problem is described by the well-known Navier-Stokes equations:

$$
\begin{cases}\operatorname{div}(\boldsymbol{u} \otimes \boldsymbol{u})-\operatorname{div}\left(2 v(\boldsymbol{\mu}) \nabla^{s} \boldsymbol{u}\right)=-\nabla p, & \text { in } \Omega, \\ \operatorname{div} \boldsymbol{u}=\mathbf{0}, & \text { in } \Omega, \\ \boldsymbol{u}(x)=(1,0), & \text { on } \Gamma_{\mathrm{TOP}}, \\ \boldsymbol{u}(x)=\mathbf{0}, & \text { on } \Gamma_{0} .\end{cases}
$$

The physical problem is the classical benchmark of the lid-driven cavity problem with a parameterized diffusivity constant $v(\boldsymbol{\mu})$. In this case the impossibility of recovering an affine decomposition of the differential operators is given by the convective term, which is by nature a nonlinear term, and by the parameterized diffusion term. The diffusivity constant $v(\boldsymbol{\mu})$ has in fact been parameterized by the following nonlinear function:

$$
v(\boldsymbol{x} ; \boldsymbol{\mu})=\frac{e^{2\left(-2\left(x_{1}-\mu_{1}-0.5\right)^{2}-2\left(x_{2}-\mu_{2}-0.5\right)^{2}\right)}}{100}+0.01,
$$

which is a Gaussian function and the position of whose center has been parameterized using the parameter vector $\boldsymbol{\mu}=\left(\mu_{1}, \mu_{2}\right)$. For the particular case, the discretized algebraic version of the continuous formulation can be rewritten as

$$
\left(\begin{array}{cc}
\boldsymbol{C}(\boldsymbol{u})+\boldsymbol{A}(\boldsymbol{\mu}) & \boldsymbol{B}^{T} \\
\boldsymbol{B} & 0
\end{array}\right)\left(\begin{array}{l}
\boldsymbol{u} \\
p
\end{array}\right)=\left(\begin{array}{l}
\boldsymbol{f} \\
0
\end{array}\right) .
$$

The matrix $\boldsymbol{A}(\boldsymbol{\mu})$ represents the discretized diffusion operator, the matrix $\boldsymbol{C}(\boldsymbol{u})$ represents the discretized nonlinear convective operator, while the term $\boldsymbol{B}$ represents the divergence operator. The term $\boldsymbol{A}(\boldsymbol{\mu})$ is characterized by a nonaffine parametric dependency while the term $\boldsymbol{C}(\boldsymbol{u})$ is characterized by nonlinearity with respect to the solution. The velocity and pressure fields are approximated as

$$
\boldsymbol{u}(\boldsymbol{\mu}) \approx \sum_{q=1}^{N_{u}} c_{q}^{u}(\boldsymbol{\mu}) \boldsymbol{h}_{q}^{u}, \quad p(\boldsymbol{\mu}) \approx \sum_{q=1}^{N_{p}} c_{q}^{p}(\boldsymbol{\mu}) \boldsymbol{h}_{q}^{p},
$$

and, in order to achieve an efficient offline-online splitting, the discretized operators are approximated by the matrix version of the DEIM algorithm and expressed as

$$
\boldsymbol{A}(\boldsymbol{\mu}) \approx \sum_{q=1}^{N_{A}} c_{q}^{A}(\boldsymbol{\mu}) \boldsymbol{h}_{q}^{A}, \quad \boldsymbol{C}(\boldsymbol{u}) \approx \sum_{q=1}^{N_{C}} c_{q}^{C}\left(\boldsymbol{c}_{u}\right) \boldsymbol{h}_{q}^{C} .
$$


The problem is discretized using the finite volume method and a staggered Cartesian grid made of $20 \times 20$ cell-centered finite volume elements. The DEIM algorithm has been implemented using 100 samples chosen randomly inside the training space $\mathcal{P}_{\text {EIM }}^{\text {train }} \in[-0.5,0.5]^{2}$. The magic points necessary for the implementation of the DEIM algorithm are chosen to be coincident with the cell centers of the discretized problem. The basis functions $\boldsymbol{h}_{q}^{A}$ and $\boldsymbol{h}_{C}^{A}$ are obtained using the DEIM algorithm applied on the vectorized version of the discretized differential operator snapshots computed during the training stage $\boldsymbol{S}_{A}=\left[\operatorname{vec}\left(\boldsymbol{A}_{1}\right), \ldots, \operatorname{vec}\left(\boldsymbol{A}_{M}\right)\right]$ and $\boldsymbol{S}_{C}=\left[\operatorname{vec}\left(\boldsymbol{C}_{1}\right), \ldots, \operatorname{vec}\left(\boldsymbol{C}_{M}\right)\right]$. The snapshot matrices $\boldsymbol{S}_{A}$ and $\boldsymbol{S}_{C}$ contain in fact the discretized differential operators in vector form obtained for the different samples of the training set.

In Figure 1.10 we report the comparison of the full-order model fields and the ROM ones; the comparison is depicted for a parameter sample not used to train the ROM.

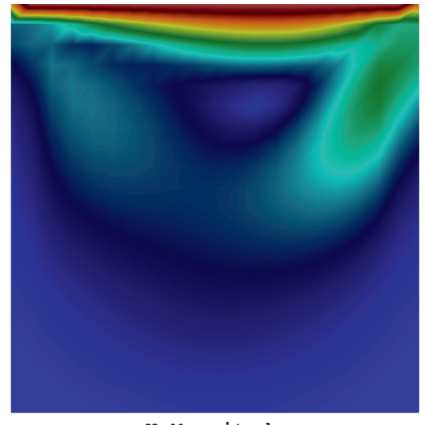

U Magnitude

$0.00 \mathrm{e}+000.20 .30 .40 .00$

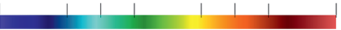

(a)

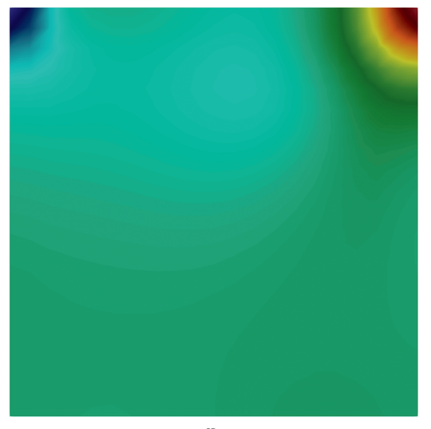

$-4.20 e-01$



Ured Magnitude

$0.00 \mathrm{e}+000.20 .30 .40 .50 .60 .70 .81 .00 \mathrm{e}+00$

(b)

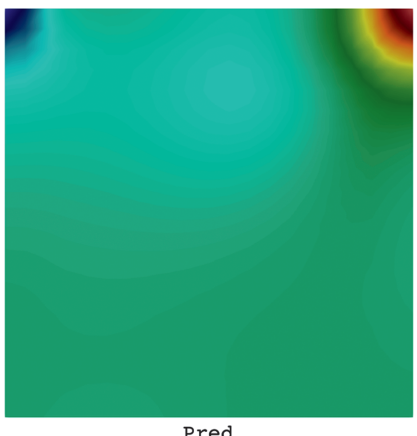

$\begin{array}{rrr}0.2 & 0.4 & 7.70 \mathrm{e}-01\end{array}$

(c)

(d)

Figure 1.10: Comparison between the FOM velocity (a) and pressure (c) fields and the ROM velocity (b) and pressure (d) fields. The plots are reported for one selected sample value inside the testing set. The ROM solutions have been computed using 14 basis functions for the velocity space, 10 for the pressure space, 10 DEIM basis functions for the convective matrix $C$, and $10 \mathrm{DEIM}$ basis functions for the diffusion matrix $\boldsymbol{B}$. 

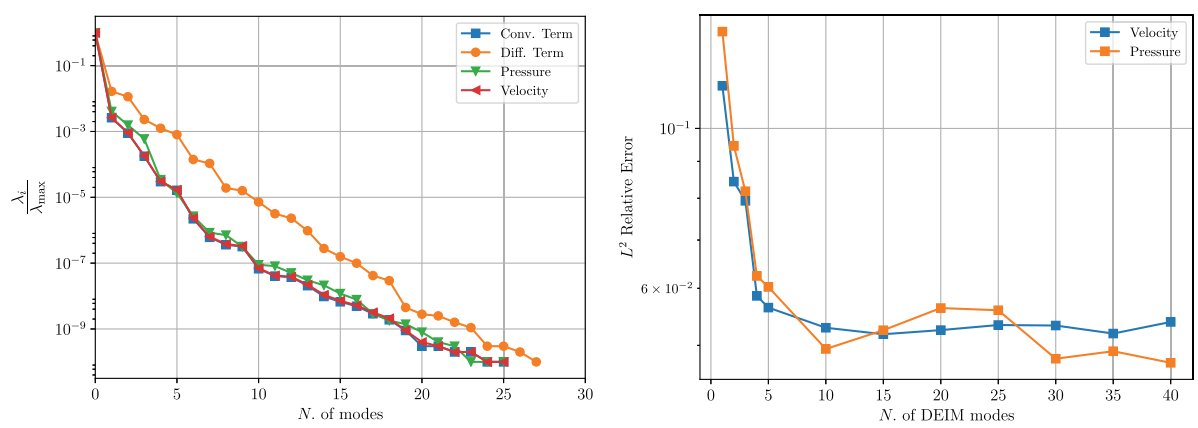

Figure 1.11: Eigenvalue decay of the POD procedure during the DEIM algorithm (left plot). The convergence analysis with respect to the number of DEIM basis functions (right plot), which is computed using the average value over the testing set of the $L^{2}$ relative error, has been performed keeping constant the number of basis functions used to approximate the velocity and pressure fields $\left(N_{u}=14, N_{p}=10\right)$ and changing the number of DEIM basis functions used to approximate the convective and diffusion terms $\left(N_{C}=N_{A}\right)$.

On the right side of Figure 1.11 we report the convergence analysis for the numerical example. The plots are performed testing the ROM on 100 additional sample values selected randomly inside the parameter space $\mathcal{P}_{\mathrm{EIM}}^{\text {test }} \in[-0.5,0.5]^{2}$. In the plots is reported the average value over the testing space of the $L^{2}$ relative error.

\subsection{Advanced tools: reduction in parameter spaces}

Often the use of the aforementioned geometrical morphing techniques in Section 1.2 does not tell us how many control points, i. e., geometrical parameters, are enough to conduct a proper analysis. This leads to self-imposing too few parameters in order to avoid the curse of dimensionality and dealing with intractable problems. To overcome this issue there exist techniques for parameter space dimensionality reduction, both linear and nonlinear. In particular we present here the active subspaces property for linear dimensionality reduction, while in the last section we show an overview of possible nonlinear methods.

These methods are intended as general tools, not restricted to parameterized PDEs. Moreover, the nature of the parameter space can be very diverse, including both geometrical and physical parameters. They are data-driven tools working with couples of input/output data, and they can be used to enhance other MOR techniques.

\subsubsection{Active subspaces property and its applications}

In this and the following sections we present the active subspaces (AS) property proposed by Trent Russi [89] and developed by Paul Constantine [31]. In brief, active sub- 
spaces are defined as the leading eigenspaces of the second moment matrix of the function's gradient and constitute a global sensitivity index.

We present how to exploit AS to reduce the parameter space dimensionality, and use it as a powerful preprocessing tool. Moreover, we show how to combine it with a model reduction methodology and present its application to a cardiovascular problem. In particular, after identifying a lower-dimensional parameter subspace, we sample it to apply further MOR methods. This results in improved computational efficiency.

The main characteristic of AS is the fact that it uses information of both the output function of interest and the input parameter space in order to reduce its dimensionality. The active subspaces have been successfully employed in many engineering fields. We cite, among others, applications in magnetohydrodynamics power generation modeling in [50], in naval engineering for the computation of the total drag resistance with both geometrical and physical parameters in [101,38], and in constrained shape optimization [66] using the concept of shared active subspaces in [100]. There are also applications to turbomachinery in [7], to uncertainty quantification in the numerical simulation of a scramjet in [34], and to the acceleration of Markov chain Monte Carlo in [35]. Extension of active subspace discovery for time-dependent processes and application to a lithium ion battery model can be found in [32]. A multifidelity approach to reduce the cost of performing dimension reduction through the computation of the active subspace matrix is presented in [62]. In [46] the authors exploit AS for Bayesian optimization, while the coupling with ROMs can be found in [39] for a nonintrusive data-driven approach, and the coupling with POD-Galerkin methods for biomedical engineering will be presented in Section 1.4.4 following [99].

\subsubsection{Active subspaces definition}

Given a parametric scalar function $f(\boldsymbol{\mu}): \mathbb{R}^{p} \rightarrow \mathbb{R}$, where $p$ is the number of parameters representing the output of interest, and given a probability density function $\rho: \mathbb{R}^{p} \rightarrow \mathbb{R}^{+}$that represents uncertainty in the model inputs, active subspaces are low-dimensional subspaces of the input space where $f$ varies the most on average. It is a property of the pair $(f, \rho)$ [31]. In order to uncover AS we exploit the gradients of the function with respect to the input parameters, so it can be viewed as a derivativebased sensitivity analysis that unveils low-dimensional parameterization of $f$ using some linear combinations of the original parameters. Roughly speaking, after a rescaling of the input parameter space to the hypercube $[-1,1]^{p}$, we rotate it until the lowerrank approximation of the output of interest is discovered, which means a preferred direction in the input space is identified. Then we can project all the data onto the orthogonal space of this preferred direction and we can construct a surrogate model on this low-dimensional space. 
Let us add some hypotheses to $f$ in order to properly construct the matrix we will use to find the active subspaces: Let $f$ be continuous and differentiable with squareintegrable partial derivatives in the support of $\rho$. We define the so-called uncentered covariance matrix $\mathbf{C}$ of the gradients of $f$ as the matrix whose elements are the average products of partial derivatives of the $\operatorname{map} f$, that is,

$$
\mathbf{C}=\mathbb{E}\left[\nabla_{\boldsymbol{\mu}} f \nabla_{\boldsymbol{\mu}} f^{T}\right]=\int\left(\nabla_{\boldsymbol{\mu}} f\right)\left(\nabla_{\boldsymbol{\mu}} f\right)^{T} \rho d \boldsymbol{\mu},
$$

where $\mathbb{E}$ is the expected value and $\nabla_{\boldsymbol{\mu}} f=\nabla f(\boldsymbol{\mu})=\left[\frac{\partial f}{\partial \mu_{1}}, \ldots, \frac{\partial f}{\partial \mu_{p}}\right]^{T}$ is the column vector of partial derivatives of $f$. This matrix is symmetric so it has a real eigenvalue decomposition:

$$
\mathbf{C}=\mathbf{W} \boldsymbol{\Lambda} \mathbf{W}^{T}
$$

where $\mathbf{W} \in \mathbb{R}^{p \times p}$ is the orthogonal matrix of eigenvectors and $\boldsymbol{\Lambda}$ is the diagonal matrix of nonnegative eigenvalues arranged in descending order. The eigenpairs of the uncentered covariance matrix define the active subspaces of the pair $(f, \rho)$. Moreover, Lemma 2.1 in [33] states that the eigenpairs are functionals of $f(\boldsymbol{\mu})$ and we have

$$
\lambda_{i}=\mathbf{w}_{i}^{T} \mathbf{C} \mathbf{w}_{i}=\int\left(\nabla_{\boldsymbol{\mu}} f^{T} \mathbf{w}_{i}\right)^{2} \rho d \boldsymbol{\mu},
$$

which means that the $i$-th eigenvalue is the average squared directional derivative of $f$ along the eigenvector $\mathbf{w}_{i}$. Alternatively we can say that the eigenvalues represent the magnitude of the variance of $\nabla_{\mu} f$ along their eigenvectors orientations. So small values of the eigenvalues correspond to small perturbation of $f$ along the corresponding eigenvectors. It also follows that large gaps between eigenvalues indicate directions where $f$ changes the most on average. Since we consider the lower-dimensional space of dimension $M<p$ where the target function has exactly this property, we define the active subspace of dimension $M$ as the span of the first $M$ eigenvectors (they correspond to the most energetic eigenvalues before a gap). Let us partition $\boldsymbol{\Lambda}$ and $\mathbf{W}$ as

$$
\boldsymbol{\Lambda}=\left[\begin{array}{ll}
\boldsymbol{\Lambda}_{1} & \\
& \boldsymbol{\Lambda}_{2}
\end{array}\right], \quad \mathbf{W}=\left[\begin{array}{ll}
\mathbf{W}_{1} & \mathbf{W}_{2}
\end{array}\right],
$$

where $\boldsymbol{\Lambda}_{1}=\operatorname{diag}\left(\lambda_{1}, \ldots, \lambda_{M}\right)$ and $\mathbf{W}_{1}$ contains the first $M$ eigenvectors. We can use $\mathbf{W}_{1}$ to project the original parameters to the active subspace obtaining the reduced parameters, that is, the input space is geometrically transformed and aligned with $\mathbf{W}_{1}$, in order to retain only the directions where the function variability is high. We call the active variable $\boldsymbol{\mu}_{M}$ the range of $\mathbf{W}_{1}^{T}$ and the inactive variable $\boldsymbol{\eta}$ the range of $\mathbf{W}_{2}^{T}$ :

$$
\boldsymbol{\mu}_{M}=\mathbf{W}_{1}^{T} \boldsymbol{\mu} \in \mathbb{R}^{M}, \quad \boldsymbol{\eta}=\mathbf{W}_{2}^{T} \boldsymbol{\mu} \in \mathbb{R}^{p-M} .
$$

We can thus express any point in the parameter space $\boldsymbol{\mu} \in \mathbb{R}^{p}$ in terms of $\boldsymbol{\mu}_{M}$ and $\boldsymbol{\eta}$ as

$$
\boldsymbol{\mu}=\mathbf{W} \mathbf{W}^{T} \boldsymbol{\mu}=\mathbf{W}_{1} \mathbf{W}_{1}^{T} \boldsymbol{\mu}+\mathbf{W}_{2} \mathbf{W}_{2}^{T} \boldsymbol{\mu}=\mathbf{W}_{1} \boldsymbol{\mu}_{M}+\mathbf{W}_{2} \boldsymbol{\eta} .
$$


The lower-dimensional approximation, or surrogate quantity of interest, $g$ : $\mathbb{R}^{M} \rightarrow \mathbb{R}$ of the target function $f$ is a function of only the active variable $\boldsymbol{\mu}_{M}$ as

$$
f(\boldsymbol{\mu}) \approx g\left(\mathbf{W}_{1}^{T} \boldsymbol{\mu}\right)=g\left(\boldsymbol{\mu}_{M}\right)
$$

Such $g$ is called ridge function [77] and, as we can infer from this section, it is constant along the span of $\mathbf{W}_{2}$.

From a practical point of view, equation (1.92) is estimated through the Monte Carlo method. We draw $N_{\text {train }}$ independent samples $\boldsymbol{\mu}^{(i)}$ according to the measure $\rho$ and we approximate

$$
\mathbf{C} \approx \hat{\mathbf{C}}=\frac{1}{N_{\text {train }}} \sum_{i=1}^{N_{\text {train }}} \nabla_{\boldsymbol{\mu}} f_{i} \nabla_{\boldsymbol{\mu}} f_{i}^{T}=\hat{\mathbf{W}} \hat{\mathbf{\Lambda}} \hat{\mathbf{W}}^{T},
$$

where $\nabla_{\boldsymbol{\mu}} f_{i}=\nabla_{\boldsymbol{\mu}} f\left(\boldsymbol{\mu}^{(i)}\right)$. In [31] the authors provide a heuristic formula for the number of samples $N_{\text {train }}$ needed to properly estimate the first $k$ eigenvalues, that is,

$$
N_{\text {train }}=\alpha k \ln (p) \text {, }
$$

where $\alpha$ usually is between 2 and 10. Moreover, they prove that for sufficiently large $N_{\text {train }}$ the error $\varepsilon$ committed in the approximation of the active subspace of dimension $n$ is bounded from above by

$$
\varepsilon=\operatorname{dist}\left(\operatorname{rank}\left(\mathbf{W}_{1}\right), \operatorname{rank}\left(\hat{\mathbf{W}}_{1}\right)\right) \leq \frac{4 \lambda_{1} \delta}{\lambda_{n}-\lambda_{n+1}},
$$

where $\delta$ is a positive scalar bounded from above by $\frac{\lambda_{n}-\lambda_{n+1}}{5 \lambda_{1}}$. Here we can clearly see how the gap between two eigenvalues is important in order to properly approximate $f$ exploiting AS.

\subsubsection{Some examples}

In this section we present two simple examples with the computation of the active subspaces using analytical gradients. To highlight the possibility that the presence of an active subspace is not always guaranteed we also show an example in this direction. We choose for both the cases a three-dimensional input parameter space without loss of generality. In order to identify the low-dimensional structure of the function of interest we use the sufficient summary plots, developed in [36]. In our cases, they are scatter plots of $f(\boldsymbol{\mu})$ against the active variable $\boldsymbol{\mu}_{M}$.

The presence of an active subspace is not always guaranteed. For example, not every target function that has a radial symmetry has a lower-dimensional representation in terms of active variables. This is due to the fact that there is no rotation of the 
input parameter space that aligns it along a preferred direction since all of them are equally important.

Let us consider for example the function $f(\boldsymbol{\mu})=\frac{1}{2} \boldsymbol{\mu}^{T} \boldsymbol{\mu}$ representing an $n$-dimensional elliptic paraboloid, where the parameter $\boldsymbol{\mu}$ is a column vector in $[-1,1]^{3}$. In this case we have the exact derivatives, in fact, $\nabla_{\boldsymbol{\mu}} f=\boldsymbol{\mu}$, and we do not have to approximate them. If we draw 1,000 samples and we apply the procedure to find an active subspace and we plot the sufficient summary plot in one dimension, as in Figure 1.12, we clearly see how it is unable to find the active variable along which $f$ varies the most on average. In fact there is not a significant gap between the eigenvalues, since we have $\mathbf{C}=\frac{1}{3}$ Id. Moreover the projection of the data onto the inactive subspace suggests the presence of an $n$-dimensional elliptic paraboloid.
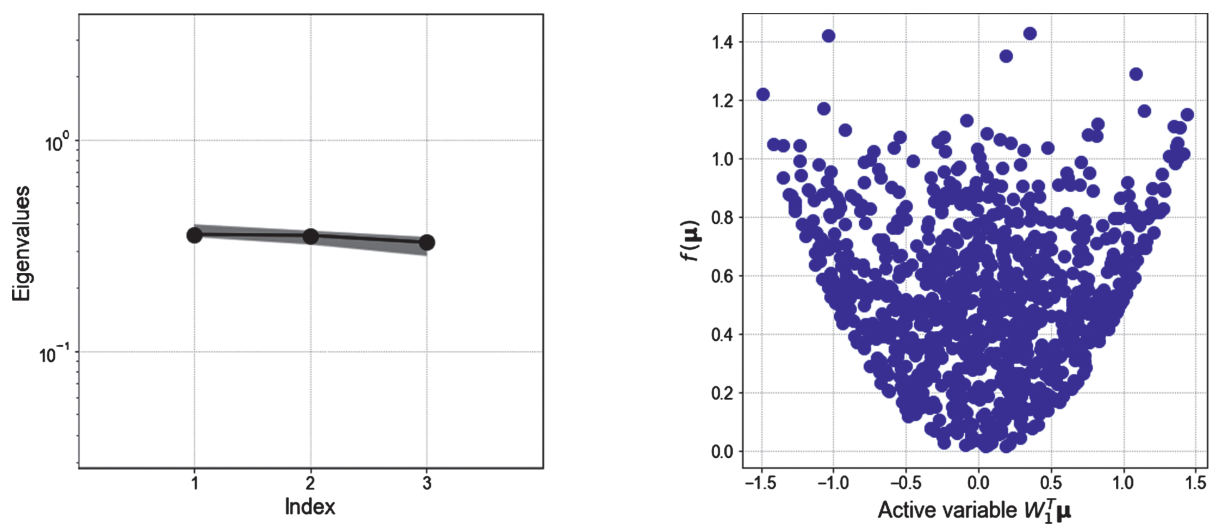

Figure 1.12: Example of an output function with a radial symmetry. On the left the exact eigenvalues of the uncentered covariance matrix are shown. On the right the sufficient summary plot in one dimension $\left(f(\boldsymbol{\mu})\right.$ against $\boldsymbol{\mu}_{M}=\mathbf{W}_{1}^{T} \boldsymbol{\mu}$ ) shows how the projection of the data along the inactive directions does not unveil a lower-dimensional structure for $f$.

Let us consider now another quadratic function in three variables. We define the output of interest $f$ as

$$
f(\boldsymbol{\mu})=\frac{1}{2} \boldsymbol{\mu}^{T} \mathbf{A} \boldsymbol{\mu}
$$

where $\boldsymbol{\mu} \in[-1,1]^{3}$ and $\mathbf{A}$ is symmetric positive definite with a major gap between the first and the second eigenvalue. With this form we can compute the exact gradients as $\nabla_{\boldsymbol{\mu}} f(\boldsymbol{\mu})=\mathbf{A} \boldsymbol{\mu}$ and, taking $\rho$ as a uniform density function, compute $\mathbf{C}$ as

$$
\mathbf{C}=\mathbf{A}\left(\int \boldsymbol{\mu} \boldsymbol{\mu}^{T} \rho d \boldsymbol{\mu}\right) \mathbf{A}^{T}=\frac{1}{3} \mathbf{A}^{2}
$$


So the squared eigenvalues of $\mathbf{A}$ are the eigenvalues of $\mathbf{C}$. Since, by definition, $\mathbf{A}$ has a significant gap between the first and second eigenvalues, we can easily find an active subspace of dimension one.

In Figure 1.13 we show the sufficient summary plot of $f$ with respect to its active variable. A clear univariate behavior is present, as expected, so we can easily construct $g$, for instance taking a quadratic one-dimensional function. We can also see the associated eigenvalues of the uncentered covariance matrix.
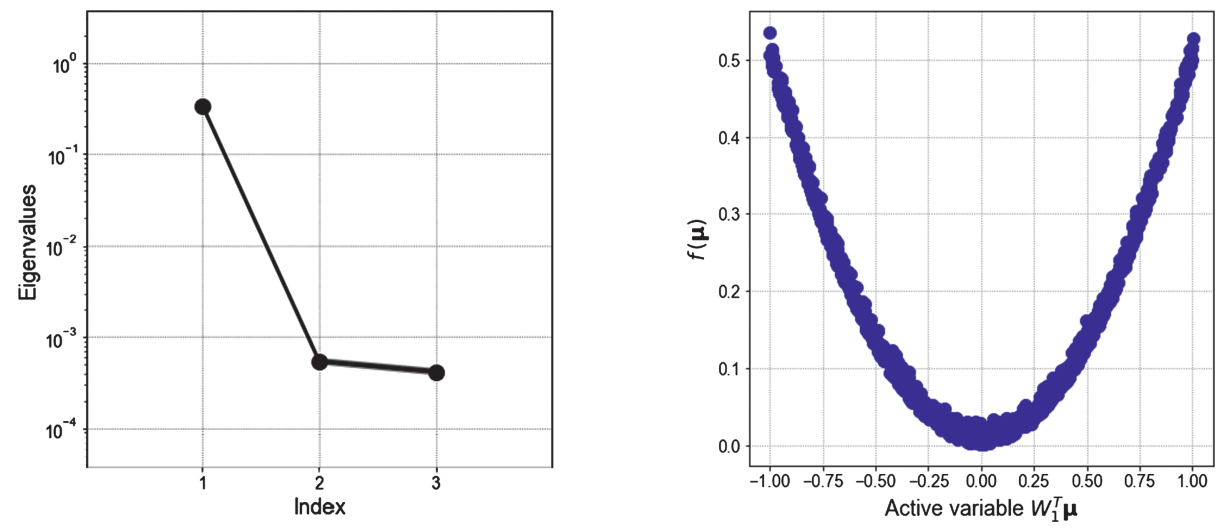

Figure 1.13: Example of a quadratic function with an active subspace of dimension one. On the left the exact eigenvalues of the uncentered covariance matrix are shown. On the right the sufficient summary plot in one dimension $\left(f(\boldsymbol{\mu})\right.$ against $\left.\boldsymbol{\mu}_{M}=\mathbf{W}_{1}^{T} \boldsymbol{\mu}\right)$ shows how the projection of the data along the inactive directions unveils a univariate structure for $f$.

\subsubsection{Active subspaces as preprocessing tool to enhance model reduction}

The presence of an active subspace for an output of interest, derived from the solution of a parametric PDE, can be exploited for further MOR. Thus, in this context, AS can be seen as a powerful preprocessing technique to both reduce the parameter space dimensionality and boost the performance of other model order reduction methods.

In [99] the active subspace for a relative pressure drop in a stenosed carotid artery is used as a reduced sampling space to improve the reconstruction of the output manifold. We used as parameters the displacement of a selection of RBF control points to simulate the occlusion of the carotid artery after the bifurcation. For a review of the RBF interpolation technique, see Section 1.2.2. In Figure 1.5 two different views of the same carotid are shown and the control points are highlighted with green dots. The target function was a relative pressure drop between the two branches computed solving a stationary Navier-Stokes problem. 
After the identification of the active subspace we exploit it by sampling the original full parameter space along the active subspace. These sampled parameters were used, in the offline phase, to construct the snapshots matrix for the training of an ROM. This leads to better approximation properties for a given number of snapshots with respect to usual sampling techniques. The natural construction of the uncentered covariance matrix, which uses information from both the inputs and the outputs, is the reason of such improvements.

The same idea has been coupled also with nonintrusive MOR techniques, such as POD with interpolation (PODI), in [102], while for the reconstruction of modal coefficients using PODI with AS for low computational budgets we suggest [39].

\subsubsection{About nonlinear dimensionality reduction}

There are plenty of other techniques that reduce the dimensionality of a given data set. They do not exploit simultaneously the structure of the output function and the input parameter space like AS, they just express the data vectors we want to reduce in a reduced space embedded in the original one. For a comprehensive overview, see [64] and [104]. The main assumption is that the data set at hand has an intrinsic dimensionality, which is lower than that of the full space where they belong. This means that the data are lying on or near a manifold with dimensionality $d$ embedded in a greater space of dimension $D$. If we approximate this manifold with a linear subspace we use a linear dimensionality reduction technique; otherwise assuming the data lie on a curved manifold we can achieve better results using a nonlinear method. Unfortunately in general neither the characteristics of the manifold, nor the intrinsic dimensionality are known, so the dimensionality reduction problem is ill-posed. There are several algorithms to detect the intrinsic dimensionality of a data set; we suggest the review in [17]. Among all we cite two of the most popular techniques, i.e., locally linear embedding (LLE), presented in [83], and Isomap [98]. Extensions for the two methods can be found in [12].

LLE seeks to preserve local properties of the high-dimensional data in the embedded space, and it is able to detect nonconvex manifolds. In particular it preserves local reconstruction weights of the neighborhood graph, that is, LLE fits a hyperplane through each data point and its nearest neighbors. Some applications can be found in [51] for biomedical engineering, or in [60] for computational mechanics.

Isomap instead seeks to preserve geodesic (or curvilinear) distances between the high-dimensional data points and the lower-dimensional embedded ones. Its topological stability has been investigated in [8], while it has been used for micromotility reconstruction in [5].

Other approaches include for example a manifold walking algorithm that has been proposed in [73] and in [74]. 


\subsection{Conclusion and outlook}

This introductory chapter provided the means to understand projection-based MOR methods in Section 1.1. Various techniques allowing the parameterization of complicated geometries were provided in Section 1.2. Since many geometries of interest introduce nonlinearities or nonaffine parameter dependency, an intermediate step such as the EIM is often applied. The basics were presented in Section 1.3 and will be used further in the chapter on hyperreduction (Chapter 5) of this volume. The reduction in parameter space becomes necessary if high-dimensional parameter spaces are considered. Active subspaces (Section 1.4) provide a mean to tackle the curse of dimensionality.

Each chapter of the handbook gives in-depth technical details upon a particular topic of interest. This includes common MOR methods, several application areas of interest, and a survey of current software frameworks for model reduction. Whenever a method does not rely only on the PDE-based functional analysis setting introduced in this chapter, corresponding requirements will be mentioned within each technical chapter.

\section{Bibliography}

[1] R. A. Adams, Sobolev Spaces, Academic Press New York, 1975.

[2] M. Ainsworth and J. T. Oden, A posteriori error estimation in finite element analysis, Computer Methods in Applied Mechanics and Engineering, 142 (1) (1997), 1-88.

[3] B. O. Almroth, P. Stern and F. A. Brogan, Automatic choice of global shape functions in structural analysis, AIAA Journal, 16 (5) (1978), 525-528.

[4] D. Arnold et al., Unified analysis of discontinuous Galerkin methods for elliptic problems, SIAM Journal on Numerical Analysis, 39 (5) (2002), 1749-1779.

[5] M. Arroyo et al., Reverse engineering the euglenoid movement, Proceedings of the National Academy of Sciences, 109 (44) (2012), 17874-17879.

[6] I. Babuska, Error-bounds for finite element method, Numerische Mathematik, 16 (1970/1971), 322-333.

[7] S. Bahamonde et al., Active subspaces for the optimal meanline design of unconventional turbomachinery, Applied Thermal Engineering, 127 (2017), 1108-1118.

[8] M. Balasubramanian and E. L. Schwartz, The isomap algorithm and topological stability, Science, 295 (5552) (2002), 7.

[9] F. Ballarin et al., A POD-selective inverse distance weighting method for fast parametrized shape morphing, International Journal for Numerical Methods in Engineering, 117 (8) (2019), 860-884, 10.1002/nme.5982.

[10] M. Barrault et al., An 'empirical interpolation' method: application to efficient reduced-basis discretization of partial differential equations, Comptes Rendus. Mathématique, 339 (9) (2004), 667-672, 10.1016/j.crma.2004.08.006.

[11] A. Beckert and H. Wendland, Multivariate interpolation for fluid-structure-interaction problems using radial basis functions, Aerospace Science and Technology, 5 (2) (2001), 125-134. 
[12] Y. Bengio et al., Out-of-sample extensions for lle, isomap, mds, eigenmaps, and spectral clustering, in Advances in Neural Information Processing Systems, pp. 177-184, 2004.

[13] D. Boffi, F. Brezzi and M. Fortin, Mixed Finite Element Methods and Applications, Springer-Verlag Berlin Heidelberg, 2013.

[14] D. Bonomi, A. Manzoni and A. Quarteroni, A matrix DEIM technique for model reduction of nonlinear parametrized problems in cardiac mechanics, Computer Methods in Applied Mechanics and Engineering, 324 (2017), 300-326, 10.1016/j.cma.2017.06.011.

[15] M. D. Buhmann, Radial Basis Functions: Theory and Implementations, vol. 12, Cambridge university press, 2003.

[16] T. Bui-Thanh, M. Damodaran and K. Willcox, Proper orthogonal decomposition extensions for parametric applications in compressible aerodynamics, in 21st AIAA Applied Aerodynamics Conference, American Institute of Aeronautics and Astronautics, jun 2003, 10.2514/6.2003-4213.

[17] F. Camastra, Data dimensionality estimation methods: a survey, Pattern Recognition, 36 (12) (2003), 2945-2954.

[18] C. Canuto, T. Tonn and K. Urban, A posteriori error analysis of the reduced basis method for nonaffine parametrized nonlinear PDEs, SIAM Journal on Numerical Analysis, 47 (3) (2009), 2001-2022, 10.1137/080724812.

[19] C. Canuto et al., Spectral Methods: Fundamentals in Single Domains, Springer-Verlag Berlin Heidelberg, 2006.

[20] K. Carlberg, C. Bou-Mosleh and C. Farhat, Efficient non-linear model reduction via a least-squares Petrov-Galerkin projection and compressive tensor approximations, International Journal for Numerical Methods in Engineering, 86 (2) (2010), 155-181, 10.1002/nme.3050.

[21] F. Casenave, A. Ern and T. Lelièvre, A nonintrusive reduced basis method applied to aeroacoustic simulations, Advances in Computational Mathematics, 41 (5) (2014), 961-986, 10.1007/s10444-014-9365-0.

[22] S. Chaturantabut and D.C. Sorensen, Discrete empirical interpolation for nonlinear model reduction, in:Proceedings of the 48h IEEE Conference on Decision and Control (CDC) held jointly with 2009 28th Chinese Control Conference, IEEE, dec 2009, 10.1109/cdc.2009.5400045.

[23] S. Chaturantabut and D. C. Sorensen, Nonlinear model reduction via discrete empirical interpolation, SIAM Journal on Scientific Computing, 32 (5) (2010), 2737-2764, $10.1137 / 090766498$.

[24] P. Chen, A. Quarteroni and G. Rozza, A weighted empirical interpolation method: a priori convergence analysis and applications, ESAIM: Mathematical Modelling and Numerical Analysis, 48 (4) (2014), 943-953, 10.1051/m2an/2013128.

[25] Y. Chen, A certified natural-norm successive constraint method for parametric inf-sup lower bounds, Applied Numerical Mathematics, 99 (2016), 98-108, ISSN: 0168-9274.

[26] Y. Chen et al., A monotonic evaluation of lower bounds for inf-sup stability constants in the frame of reduced basis approximations, Comptes Rendus. Mathématique, 346 (23) (2008), 1295-1300.

[27] Y. Chen et al., Improved successive constraint method based a posteriori error estimate for reduced basis approximation of 2D Maxwell's problem, ESAIM: Mathematical Modelling and Numerical Analysis, 43 (6) (2009), 1099-1116.

[28] F. Chinesta et al., Model order reduction, in Encyclopedia of Computational Mechanics, Second Edition, pp.1-36, Elsevier, 2017.

[29] P. Ciarlet, The Finite Element Method for Elliptic Problems, Classics in Applied Mathematics, vol. 40, Society for Industrial and Applied Mathematics, Philadelphia, 2002. 
[30] P. Ciarlet, Linear and Nonlinear Functional Analysis with Applications, Society for Industrial and Applied Mathematics, Philadelphia, 2014.

[31] P. G. Constantine, Active Subspaces: Emerging Ideas for Dimension Reduction in Parameter Studies, vol. 2, SIAM, 2015.

[32] P. G. Constantine and A. Doostan, Time-dependent global sensitivity analysis with active subspaces for a lithium ion battery model, Statistical Analysis and Data Mining: The ASA Data Science Journal, 10 (5) (2017), 243-262.

[33] P. G. Constantine, E. Dow and Q. Wang, Active subspace methods in theory and practice: applications to kriging surfaces, SIAM Journal on Scientific Computing, 36 (4) (2014), A1500-A1524.

[34] P. G. Constantine, et al., Exploiting active subspaces to quantify uncertainty in the numerical simulation of the HyShot II scramjet, Journal of Computational Physics, 302 (2015), 1-20.

[35] P. G. Constantine, C. Kent and T. Bui-Thanh, Accelerating Markov chain Monte Carlo with active subspaces, SIAM Journal on Scientific Computing, 38 (5) (2016), A2779-A2805.

[36] R. D. Cook, Regression Graphics: Ideas for Studying Regressions through Graphics, vol. 482, John Wiley \& Sons, 2009.

[37] N. Demo et al., Shape optimization by means of proper orthogonal decomposition and dynamic mode decomposition, in Technology and Science for the Ships of the Future: Proceedings of NAV 2018: 19th International Conference on Ship \& Maritime Research, pp. 212-219, IOS Press, 2018, 10.3233/978-1-61499-8709-212.

[38] N. Demo et al., An efficient shape parametrisation by free-form deformation enhanced by active subspace for hull hydrodynamic ship design problems in open source environment, in The 28th International Ocean and Polar Engineering Conference, 2018.

[39] N. Demo, M. Tezzele and G. Rozza, A non-intrusive approach for proper orthogonal decomposition modal coefficients reconstruction through active subspaces, Comptes Rendus de l'Académie des Sciences DataBEST 2019 (Special Issue) (2019).

[40] S. Deparis, D. Forti and A. Quarteroni, A rescaled localized radial basis function interpolation on non-Cartesian and nonconforming grids, SIAM Journal on Scientific Computing, 36 (6) (2014), A2745-A2762.

[41] S. Deparis and G. Rozza, Reduced basis method for multi-parameter-dependent steady Navier-Stokes equations: applications to natural convection in a cavity, Journal of Computational Physics, 228 (12) (2009), 4359-4378.

[42] J. Duchon, Splines minimizing rotation-invariant semi-norms in Sobolev spaces, Constructive Theory of Functions of Several Variables (1977), 85-100.

[43] C. Eckart and G. Young, The approximation of one matrix by another of lower rank. Psychometrika, Psychometrika, 1(1936), 211-218, 10.1007/BF02288367.

[44] J. L. Eftang, M. A. Grepl and A. T. Patera, A posteriori error bounds for the empirical interpolation method, Comptes Rendus. Mathématique, 348 (9) (2010), 575-579, 10.1016/j.crma.2010.03.004.

[45] J. L. Eftang and B. Stamm, Parameter multi-domain 'hp' empirical interpolation, International Journal for Numerical Methods in Engineering, 90 (4) (2012), 412-428, 10.1002/nme.3327.

[46] D. Eriksson et al., Scaling Gaussian process regression with derivatives, in Advances in Neural Information Processing Systems, pp. 6867-6877, 2018.

[47] L. C. Evans, Partial Differential Equations, American Mathematical Society, 1998.

[48] R. Eymard, T. R. Gallouët and R. Herbin, The finite volume method, in P. G. Ciarlet and J. L. Lions (eds.) Handbook of Numerical Analysis, vol. 7, pp. 713-1020, 2000.

[49] D. Forti and G. Rozza, Efficient geometrical parametrisation techniques of interfaces for reduced-order modelling: application to fluid-structure interaction coupling problems, International Journal of Computational Fluid Dynamics, 28 (3-4) (2014), 158-169. 
[50] A. Glaws et al., Dimension reduction in magnetohydrodynamics power generation models: Dimensional analysis and active subspaces, Statistical Analysis and Data Mining: The ASA Data Science Journal, 10 (5) (2017), 312-325.

[51] D. González, E. Cueto and F. Chinesta, Computational patient avatars for surgery planning, Annals of Biomedical Engineering, 44 (1) (2016), 35-45.

[52] T. Graetsch and K.-J. Bathe, A posteriori error estimation techniques in practical finite element analysis, Computers \& Structures, 83 (4) (2005), 235-265.

[53] M. A. Grepl et al., Efficient reduced-basis treatment of nonaffine and nonlinear partial differential equations, ESAIM: Mathematical Modelling and Numerical Analysis, 41 (3) (2007), 575-605, 10.1051/m2an:2007031.

[54] M. W. Hess, S. Grundel and P. Benner, Estimating the inf-sup constant in reduced basis methods for time-harmonic Maxwell's equations, IEEE Transactions on Microwave Theory and Techniques, 63 (2015), 3549-3557.

[55] J. Hesthaven, B. Stamm and S. Zhang, Certified reduced basis method for the electric field integral equation, SIAM Journal on Scientific Computing, 34 (3) (2012), A1777-A1799.

[56] J.S. Hesthaven, B. Stamm and S. Zhang, Efficient greedy algorithms for high-dimensional parameter spaces with applications to empirical interpolation and reduced basis methods, ESAIM: Mathematical Modelling and Numerical Analysis, 48 (1) (2014), 259-283, 10.1051/m2an/2013100.

[57] D. B. P. Huynh et al., A successive constraint linear optimization method for lower bounds of parametric coercivity and inf-sup stability constants, Comptes Rendus. Mathématique, 345 (8) (2007), 473-478.

[58] D. B. P. Huynh et al., A natural-norm successive constraint method for inf-sup lower bounds, Computer Methods in Applied Mechanics and Engineering, 199 (29-32) (2010), 1963-1975.

[59] L. Iapichino, S. Ulbrich and S. Volkwein, Multiobjective PDE-constrained optimization using the reduced-basis method, Advances in Computational Mathematics, 43 (5) (2017), 945-972.

[60] R. Ibanez et al., A manifold learning approach to data-driven computational elasticity and inelasticity, Archives of Computational Methods in Engineering, 25 (1) (2018), 47-57.

[61] A. Kolmogoroff, Uber Die Beste Annaherung Von Funktionen Einer Gegebenen Funktionenklasse, Annals of Mathematics, 37 (1) (1936), 107, 10.2307/1968691.

[62] R. R. Lam et al., Multifidelity dimension reduction via active subspaces, SIAM Journal on Scientific Computing, 42 (2) (2020), A929-A956.

[63] T. Lassila and G. Rozza, Parametric free-form shape design with PDE models and reduced basis method, Computer Methods in Applied Mechanics and Engineering, 199 (23-24) (2010), 1583-1592.

[64] J. A. Lee and M. Verleysen, Nonlinear Dimensionality Reduction, Springer Science \& Business Media, 2007.

[65] M. Lombardi et al., Numerical simulation of sailing boats: dynamics, FSI, and shape optimization, in Variational Analysis and Aerospace Engineering: Mathematical Challenges for Aerospace Design, p. 339, Springer, 2012.

[66] T. W. Lukaczyk et al., Active subspaces for shape optimization, in 10th AIAA Multidisciplinary Design Optimization Conference, p. 1171, 2014.

[67] Y. Maday, O. Mula and G. Turinici, Convergence analysis of the generalized empirical interpolation method, SIAM Journal on Numerical Analysis, 54 (3) (2016), 1713-1731, $10.1137 / 140978843$.

[68] Y. Maday and O. Mula, A generalized empirical interpolation method: application of reduced basis techniques to data assimilation, in Analysis and Numerics of Partial Differential Equations, pp. 221-235, Springer Milan, 2013, 10.1007/978-88-470-2592-9_13. 
[69] Y. Maday et al., A general multipurpose interpolation procedure: the magic points, Communications on Pure and Applied Analysis, 8 (1) (2008), 383-404, 10.3934/cpaa.2009.8.383.

[70] A. Manzoni, An efficient computational framework for reduced basis approximation and a posteriori error estimation of parametrized Navier-Stokes flows, ESAIM: Mathematical Modelling and Numerical Analysis, 48 (4) (2014), 1199-1226.

[71] A. Manzoni and F. Negri, Heuristic strategies for the approximation of stability factors in quadratically nonlinear parametrized PDEs, Advances in Computational Mathematics, 41 (5) (2015), 1255-1288.

[72] A. Manzoni, A. Quarteroni and G. Rozza, Model reduction techniques for fast blood flow simulation in parametrized geometries, International Journal for Numerical Methods in Biomedical Engineering, 28 (6-7) (2012), 604-625.

[73] L. Meng et al., Identification of material properties using indentation test and shape manifold learning approach, Computer Methods in Applied Mechanics and Engineering, 297 (2015), 239-257.

[74] L. Meng et al., Nonlinear shape-manifold learning approach: concepts, tools and applications, Archives of Computational Methods in Engineering, 25 (1) (2018), 1-21.

[75] A. M. Morris, C. B. Allen and T. C. S. Rendall, CFD-based optimization of aerofoils using radial basis functions for domain element parameterization and mesh deformation, International Journal for Numerical Methods in Fluids, 58 (8) (2008), 827-860.

[76] A. K. Noor, On making large nonlinear problems small, Computer Methods in Applied Mechanics and Engineering, 34 (1982), 955-985.

[77] A. Pinkus, Ridge Functions, vol. 205, Cambridge University Press, 2015.

[78] C. Prud'Homme et al., Reliable real-time solution of parametrized partial differential equations: Reduced-basis output bound methods, Journal of Fluids Engineering, 124 (1) (2002), 70-80.

[79] PyGeM, Python Geometrical Morphing, 2017. https://github.com/mathLab/PyGeM (visited on 01/2017).

[80] A. Quarteroni, Numerical Models for Differential Problems, Modeling, Simulation and Applications vol. 16, Springer International Publishing, 2017.

[81] T. Rebollo et al., On a certified Smagorinsky reduced basis turbulence model, SIAM Journal on Numerical Analysis, 55 (6) (2017), 3047-3067.

[82] M. Renardy and R. C. Rogers, An Introduction to Partial Differential Equations, Springer-Verlag New York, 2004.

[83] S. T. Roweis and L. K. Saul, Nonlinear dimensionality reduction by locally linear embedding, Science, 290 (5500) (2000), 2323-2326.

[84] G. Rozza et al., Real-time reliable simulation of heat transfer phenomena, in ASME -American Society of Mechanical Engineers - Heat Transfer Summer Conference, paper HT2009-88212, volume 3, pp. 851-860, 2009.

[85] G. Rozza, D. B. P. Huynh and A. T. Patera, Reduced basis approximation and a posteriori error estimation for affinely parametrized elliptic coercive partial differential equations, Archives of Computational Methods in Engineering, 15 (2008), 229-275.

[86] G. Rozza, A. Koshakji and A. Quarteroni, Free form deformation techniques applied to 3D shape optimization problems, Communications in Applied and Industrial Mathematics, 4 (2013), 1-26, 10.1685/journal.caim.452.

[87] G. Rozza et al., Advances in reduced order methods for parametric industrial problems in computational fluid dynamics, in ECCOMAS ECCM - ECFD Conference Proceedings, Glasgow, UK, 2018. 
[88] W. Rudin, Principles of Mathematical Analysis, International Series in Pure and Applied Mathematics, McGraw-Hill, 1976.

[89] T. M. Russi, Uncertainty Quantification with Experimental Data and Complex System Models, Ph.D. thesis, UC Berkeley, 2010.

[90] F. Salmoiraghi et al., Advances in geometrical parametrization and reduced order models and methods for computational fluid dynamics problems in applied sciences and engineering: Overview and perspectives, ECCOMAS Congress 2016 - Proceedings of the 7th European Congress on Computational Methods in Applied Sciences and Engineering, 1 (2016), 1013-1031, 10.7712/100016.1867.8680.

[91] F. Salmoiraghi et al., Free-form deformation, mesh morphing and reduced-order methods: enablers for efficient aerodynamic shape optimisation, International Journal of Computational Fluid Dynamics, 32 (4-5) (2018), 233-247, 10.1080/10618562.2018.1514115.

[92] D. T. Sandwell, Biharmonic spline interpolation of GEOS-3 and SEASAT altimeter data, Geophysical Research Letters, 14 (2) (1987), 139-142.

[93] W. H. Schilders, H. A. van der Vorst and J. Rommes, Model Order Reduction: Theory, Research Aspects and Applications, Springer-Verlag Berlin Heidelberg, 2008.

[94] T. W. Sederberg and S. R. Parry, Free-form deformation of solid geometric models, in Proceedings of SIGGRAPH - Special Interest Group on GRAPHics and Interactive Techniques, pp. 151-159, SIGGRAPH, 1986.

[95] D. Shepard, A two-dimensional interpolation function for irregularly-spaced data, in Proceedings-1968 ACM National Conference, pp. 517-524, ACM, 1968.

[96] D. Sieger, S. Menzel and M. Botsch, On shape deformation techniques for simulation-based design optimization, in New Challenges in Grid Generation and Adaptivity for Scientific Computing, pp. 281-303, Springer, 2015.

[97] G. D. Smith, Numerical Solution of Partial Differential Equations: Finite Difference Methods, Clarendon Press, Oxford Applied Mathematics and Computing Science Series, 1985.

[98] J. B. Tenenbaum, V. De Silva and J. C. Langford, A global geometric framework for nonlinear dimensionality reduction, Science, 290 (5500) (2000), 2319-2323.

[99] M. Tezzele, F. Ballarin and G. Rozza, Combined parameter and model reduction of cardiovascular problems by means of active subspaces and POD-Galerkin methods, in D. Boffi et al., (eds.) Mathematical and Numerical Modeling of the Cardiovascular System and Applications, pp. 185-207, Springer International Publishing, 2018, 10.1007/978-3-319-96649-6_8.

[100] M. Tezzele et al., Model order reduction by means of active subspaces and dynamic mode decomposition for parametric hull shape design hydrodynamics, in Technology and Science for the Ships of the Future: Proceedings of NAV 2018: 19th International Conference on Ship \& Maritime Research, pp. 569-576, IOS Press, 2018, 10.3233/978-1-61499-870-9-569.

[101] M. Tezzele et al., Dimension reduction in heterogeneous parametric spaces with application to naval engineering shape design problems, Advanced Modeling and Simulation in Engineering Sciences, 5 (1) (2018), 25, ISSN: 2213-7467, 10.1186/s40323-018-0118-3.

[102] M. Tezzele, N. Demo and G. Rozza, Shape optimization through proper orthogonal decomposition with interpolation and dynamic mode decomposition enhanced by active subspaces, in Proceedings of MARINE 2019: VIII International Conference on Computational Methods in Marine Engineering, pp. 122-133, 2019.

[103] S. Vallaghé et al., A successive constraint method with minimal offline constraints for lower bounds of parametric coercivity constant, https://hal.archives-ouvertes.fr/hal-00609212, 2011.

[104] L. Van Der Maaten, E. Postma and J. Van den Herik, Dimensionality reduction: a comparative review, Journal of Machine Learning Research, 10 (2009), 66-71. 
[105] R. Verfuerth, A Posteriori Error Estimation Techniques for Finite Element Methods, Oxford Univ. Press, 2013.

[106] K. Veroy and A. T. Patera, Certified real-time solution of the parametrized steady incompressible Navier-Stokes equations: rigorous reduced-basis a posteriori error bounds, International Journal for Numerical Methods in Fluids, 47 (8-9) (2005), 773-788.

[107] K. Veroy, D. V. Rovas and A. T. Patera, A posteriori error estimation for reduced-basis approximation of parametrized elliptic coercive partial differential equations: "convex inverse" bound conditioners, ESAIM. Control, Optimisation and Calculus of Variations, 8 (2002), 1007-1028.

[108] J.A. S. Witteveen and H. Bijl, Explicit mesh deformation using inverse distance weighting interpolation, in 19th AIAA Computational Fluid Dynamics, AIAA, 2009.

[109] M. Yano, A space-time Petrov-Galerkin certified reduced basis method: application to the Boussinesq equations, SIAM Journal on Scientific Computing, 36 (1) (2014), A232-A266.

[110] K. Yosida, Functional Analysis, Springer-Verlag Berlin Heidelberg, 1995.

[111] S. Zhang, Efficient greedy algorithms for successive constraints methods with high-dimensional parameters, Tech. Report 23, http://www.dam.brown.edu/people/ shzhang/greedy_scm.pdf, 2011. 


\title{
Carmen Gräßle, Michael Hinze, and Stefan Volkwein \\ 2 Model order reduction by proper orthogonal decomposition
}

\begin{abstract}
We provide an introduction to proper orthogonal decomposition (POD) model order reduction with focus on (nonlinear) parametric partial differential equations (PDEs) and (nonlinear) time-dependent PDEs, and PDE-constrained optimization with POD surrogate models as application. We cover the relation of POD and singular value decomposition, POD from the infinite-dimensional perspective, reduction of nonlinearities, certification with a priori and a posteriori error estimates, spatial and temporal adaptivity, input dependency of the POD surrogate model, POD basis update strategies in optimal control with surrogate models, and sketch related algorithmic frameworks. The perspective of the method is demonstrated with several numerical examples.
\end{abstract}

Keywords: POD model order reduction, (discrete) empirical interpolation, adaptivity, parametric PDEs, evolutionary PDEs, certification with error analysis

MSC 2010: 35B30, 37M99, 41A05, 65K99, 93A15, 93C05

\subsection{Introduction}

Proper orthogonal decomposition (POD) is a method which comprises the essential information contained in data sets. Data sets may have their origin in various sources, like, e. g., (uncertain) measurements of geophysical processes, numerical simulations of (parameter-dependent) complex physical problems, or (dynamical) imaging. In order to illustrate the POD idea of information extraction, let $\left\{y_{1}, \ldots, y_{n}\right\} \subset \mathbb{R}^{m}$ denote a vector cloud (which here serves as our data set), where we suppose at least one of the vectors $y_{j}$ is nonzero. Let us collect the vectors $y_{j}$ in the data matrix

$$
Y=\left[y_{1}|\ldots| y_{n}\right] \in \mathbb{R}^{m \times n} .
$$

Then we have $r=\operatorname{rank} Y \in\{1, \ldots, \min (m, n)\}$. Our aim now is to find a vector $\bar{\psi} \in \mathbb{R}^{m}$ with length one which carries as much information of this vector cloud as possible. Of course, we here have to specify what information in this context means. For this

Note: We note that parts of this work have been done while the authors Michael Hinze and Carmen Gräßle were affiliated with the University of Hamburg.

\footnotetext{
Carmen Gräßle, Germany

Michael Hinze, University of Koblenz-Landau, Universitätsstr. 1, 56070 Koblenz, Germany

Stefan Volkwein, University of Konstanz, Mathematics and Statistics, Universitätsstrasse 10, 78457

Konstanz, Germany
}

Ә Open Access. ( 2021 Carmen Gräßle et al., published by De Gruyter. (cc) BY-NC-ND This work is licensed under the Creative Commons Attribution-NonCommercial-NoDerivatives 4.0 International License. 